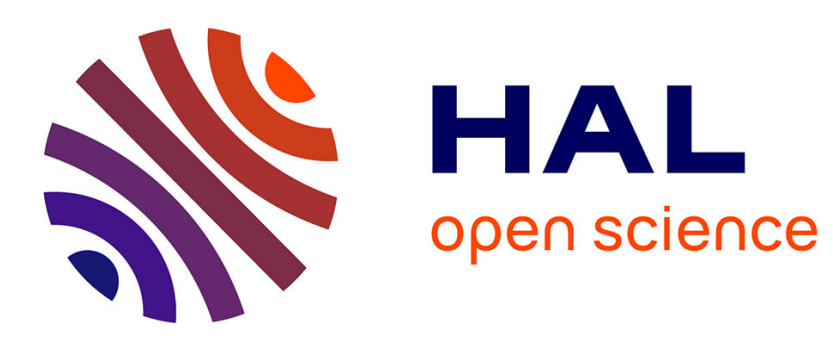

\title{
A Priori Error Estimate of a Multiscale Finite Element Method for Transport Modeling
}

Franck Ouaki, Grégoire Allaire, Sylvain Desroziers, Guillaume Enchéry

\section{To cite this version:}

Franck Ouaki, Grégoire Allaire, Sylvain Desroziers, Guillaume Enchéry. A Priori Error Estimate of a Multiscale Finite Element Method for Transport Modeling. SeMA Journal: Boletin de la Sociedad Española de Matemática Aplicada, 2015, 67 (1), pp.1-37. hal-01071637

\section{HAL Id: hal-01071637 https://hal.science/hal-01071637}

Submitted on 6 Oct 2014

HAL is a multi-disciplinary open access archive for the deposit and dissemination of scientific research documents, whether they are published or not. The documents may come from teaching and research institutions in France or abroad, or from public or private research centers.
L'archive ouverte pluridisciplinaire HAL, est destinée au dépôt et à la diffusion de documents scientifiques de niveau recherche, publiés ou non, émanant des établissements d'enseignement et de recherche français ou étrangers, des laboratoires publics ou privés. 


\title{
A PRIORI ERROR ESTIMATE OF A MULTISCALE FINITE ELEMENT METHOD FOR TRANSPORT MODELING
}

\author{
Franck Ouaki ${ }^{1}$ Grégoire Allaire ${ }^{2}$ Sylvain Desroziers ${ }^{3}$ and Guillaume Enchéry ${ }^{3}$
}

Key words. convection-diffusion, periodic homogenization, multiscale finite element method

\begin{abstract}
This work proposes an a priori error estimate of a multiscale finite element method to solve convection-diffusion problems where both velocity and diffusion coefficient exhibit strong variations at a scale which is much smaller than the domain of resolution. In that case, classical discretization methods, used at the scale of the heterogeneities, turn out to be too costly. Our method, introduced in [3], aims at solving this kind of problems on coarser grids with respect to the size of the heterogeneities by means of particular basis functions. These basis functions are defined using cell problems and are designed to reproduce the variations of the solution on an underlying fine grid. Since all cell problems are independent from each other, these problems can be solved in parallel, which makes the method very efficient when used on parallel architectures. This article focuses on the proof of an a priori error estimate of this method.
\end{abstract}

\section{Introduction.}

A multiscale finite element method was first introduced by Th.Y. Hou and X.H. Wu in [19] to efficiently solve elliptic problems with diffusion coefficients containing small-scale features. The novelty of this method consisted in computing basis functions associated to a grid with a coarser resolution than the fine scale and which contain the small-scale variations. This method was based on results of periodic homogenization theory shown, for example, in [8], [28] and [31]. Other multiscale methods, which also stem from homogenization results, were proposed in [7], [15], [23]. Since these early works, the literature has grown considerably in variety of numerical algorithms and physical applications including reservoir simulation (see e.g. the reference book $[16])$.

Here, we consider the following convection-diffusion problem in $\mathbb{R}^{N}$ (in practice $N=2$ or 3 ), written in physical units, describing the evolution of an initial concentration:

$$
\left\{\begin{array}{l}
\rho^{*}\left(x^{*}\right) \frac{\partial u^{*}}{\partial t^{*}}\left(t^{*}, x^{*}\right)+b^{*}\left(x^{*}\right) \cdot \nabla u^{*}\left(t^{*}, x^{*}\right) \\
\quad-\operatorname{div}\left(A^{*}\left(x^{*}\right) \nabla u^{*}\left(t^{*}, x^{*}\right)\right)=0, \\
u^{*}\left(0, x^{*}\right)=u^{*, 0}\left(x^{*}\right),
\end{array}\right.
$$

which arises, for example, when modeling the transport of flows in porous media. In this case, $\rho^{*}$ represents the porosity, $b^{*}$ the velocity, $A^{*}$ the diffusion tensor, $u^{*}$ the unknown concentration with initial value $u^{*, 0}$ and we assume that the flow is incompressible, i.e.,

$$
\operatorname{div}\left(b^{*}\right)=0
$$

Problem (1.1) can be adimensionalized and rescaled following the same ideas as in [1], [6] and [14], in order to be amenable to homogenization. Let $l$ be a characteristic length of the variations of the material properties, $L_{R}$ a characteristic length of macroscopic observation and $T_{R}$ a characteristic time scale. As usual, we set $\varepsilon=\frac{l}{L_{R}}$, which is assumed to be a small parameter. We denote by $\rho_{R}, b_{R}, c_{R}, A_{R}$ characteristic values for

\footnotetext{
${ }^{1}$ CEA Saclay, DEN, DMN, SRMA, Bat 453 p26D. F-91191 Gif/Yvette - France. franck.ouaki@polytechnique.edu

${ }^{2}$ CMAP UMR-CNRS 7641, École Polytechnique 91128 Palaiseau Cedex - France (G. Allaire is a member of the DEFI project at INRIA Saclay Ile-de-France) gregoire.allaire@polytechnique.edu

${ }^{3}$ IFP Energies nouvelles. 1 et 4 avenue de Bois-Préau. 92852 Rueil-Malmaison, France. e-mail: \{sylvain.desroziers, guillaume.enchery\}@ifpen.fr
} 
the porosity, velocity, concentration and diffusion and define dimensionless variables:

$$
\begin{aligned}
x & =\frac{x^{*}}{L_{R}}, t=\frac{t^{*}}{T_{R}}, \rho^{\varepsilon}(x)=\frac{\rho^{*}\left(x^{*}\right)}{\rho_{R}}, \\
b^{\varepsilon}(x) & =\frac{b^{*}\left(x^{*}\right)}{b_{R}}, A^{\varepsilon}(x)=\frac{A^{*}\left(x^{*}\right)}{A_{R}}, u_{\varepsilon}(t, x)=\frac{u^{*}\left(t^{*}, x^{*}\right)}{u_{R}} .
\end{aligned}
$$

The dimensionless equation thus reads

$$
\rho^{\varepsilon} \frac{\partial u_{\varepsilon}}{\partial t}+\frac{b_{R} T_{R}}{L_{R}} b^{\varepsilon} \cdot \nabla_{x} u_{\varepsilon}-\frac{A_{R} T_{R}}{L_{R}^{2}} \operatorname{div}_{x}\left(A^{\varepsilon} \nabla_{x} u_{\varepsilon}\right)=0
$$

We now choose a diffusive time scale, i.e., we set $T_{R}=\frac{L_{R}^{2}}{A_{R}}$. Then, for this dimensionless problem, depending on the chosen spatial scale, two Péclet numbers can be defined:

- a local one

$$
\mathbf{P e}_{l o c}=\frac{l b_{R}}{A_{R}}
$$

- and a macroscopic one

$$
\mathbf{P e}=\frac{L_{R} b_{R}}{A_{R}}
$$

They are related by $\mathbf{P e}=\frac{1}{\varepsilon} \mathbf{P} \mathbf{e}_{l o c}$. With these notations, (1.2) can be rewritten as

$$
\rho^{\varepsilon} \frac{\partial u_{\varepsilon}}{\partial t}+\mathbf{P e} b^{\varepsilon} \cdot \nabla_{x} u_{\varepsilon}-\operatorname{div}_{x}\left(A^{\varepsilon} \nabla_{x} u_{\varepsilon}\right)=0
$$

In order to keep a balance between convection and diffusion at the microscopic scale, we choose the characteristic velocity $b_{R}$ such that the local Péclet number is equal to 1 , implying that $\mathbf{P e}=\frac{1}{\varepsilon}$. Thus, the original problem (1.1) becomes:

$$
\begin{cases}\rho^{\varepsilon}(x) \frac{\partial u_{\varepsilon}}{\partial t}+\frac{1}{\varepsilon} b^{\varepsilon}(x) \cdot \nabla u_{\varepsilon}-\operatorname{div}\left(A^{\varepsilon}(x) \nabla u_{\varepsilon}\right)=0 & \text { in }(0, T) \times \mathbb{R}^{N}, \\ u_{\varepsilon}(0, x)=u^{0}(x) & \text { in } \mathbb{R}^{N},\end{cases}
$$

for some final time $T>0$. Furthermore, we shall assume that the coefficients $\rho^{\varepsilon}, b^{\varepsilon}, A^{\varepsilon}$ are $\varepsilon$-periodic functions in (1.3). The goal of the present paper is to prove an error estimate for the multiscale finite element method introduced in [3], [24] when applied to the periodic homogenization problem (1.3). Note however that our multiscale finite element method (recalled in Section 3) can be applied to non-periodic models.

In [17], a Heterogeneous Multiscale Method (HMM) was proposed and analyzed for the same problem (1.3). This HMM was designed to compute more accurately a solution at the coarse scale but not to reproduce its variation at a finer (microscopic) scale. Moreover, the analysis of this HMM assumed that the diffusion and velocity field only have a small scale behavior and that they are constant on the macroscopic scale.

In [18], Th.Y. Hou and D. Liang were concerned with the following equation:

$$
\begin{cases}\frac{\partial u_{\varepsilon}}{\partial t}+b^{\varepsilon}(x) \cdot \nabla u_{\varepsilon}-\varepsilon^{m} \operatorname{div}\left(A^{\varepsilon}(x) \nabla u_{\varepsilon}\right)=0 & \text { in }(0, T) \times \mathbb{R}^{N}, \\ u_{\varepsilon}(0, x)=u^{0}(x) & \text { in } \mathbb{R}^{N},\end{cases}
$$


where $m \in[2,+\infty[$. Our case corresponds to $m=1$ as far as the Péclet number is concerned. However the time scale is much shorter in (1.4) than in (1.3). More precisely, a time of order 1 in (1.4) is equivalent to a time of order $\varepsilon$ in (1.3).

Our paper is organized as follows. Known homogenization results for the periodic problem (1.3) are first summarized and an a priori error estimate between the exact solution and the first two terms of its two-scale expansion is recalled in Section 2. Section 3 defines our new multiscale method which is based on these previous homogenization results. Section 4 establishes an a priori error estimate of this method. Our main result is Theorem 3. Numerical tests illustrating the interest of our multiscale method can be found in the $\mathrm{PhD}$ thesis of the first author [24] and will be presented in an upcoming article.

\section{Homogenization in the periodic case.}

Let us consider the homogenization of (1.3) with periodic coefficients defined by

$$
\rho^{\varepsilon}(x)=\rho\left(\frac{x}{\varepsilon}\right), b^{\varepsilon}(x)=b\left(\frac{x}{\varepsilon}\right), A^{\varepsilon}(x)=A\left(\frac{x}{\varepsilon}\right),
$$

where $\rho, b$ and $A$ are $Y$-periodic functions, with $Y=(0,1)^{N}$ the unit cube. More precisely, we make the following assumptions.

HYPOTHESES 1.

1. $\rho, b$ and $A$ are $Y$-periodic functions,

2. $\rho, b$ and $A$ are piecewise $\mathcal{C}^{1}$ and the interfaces of discontinuity are $\mathcal{C}^{2}$,

3. $\operatorname{div}(b)=0$,

4. there exists $\rho_{\text {min }}>0$ such that $\forall y \in Y, \quad \rho(y) \geqslant \rho_{\text {min }}$,

5. A is coercive and bounded: there exist constants $C_{\text {sta }}>0$ and $C_{b n d}>0$ such that

$$
\forall y \in Y, \forall \xi \in \mathbb{R}^{N}, \quad A(y) \xi \cdot \xi \geqslant C_{s t a}|\xi|^{2},
$$

and

$$
\|A\|_{L^{\infty}(Y)^{N \times N}}=C_{b n d}<+\infty
$$

6. $u^{0} \in H^{3}\left(\mathbb{R}^{N}\right)$.

Assumption 2 is useful for getting smoothness of the cell solutions, while assumption 6 implies smoothness of the homogenized solution.

In the sequel, we denote by $X_{\#}(Y)$ the set of the $Y$-periodic functions of a given functional space $X(Y)$ (typically a Sobolev space).

The first main result of this section is the following convergence theorem which is proved in [6], [14], [22], [24] (see also [26] for a formal derivation).

TheOREM 1. Let $u_{\varepsilon}$ be the sequence of solutions to (1.3). Assuming that Hypotheses 1 are satisfied, then

$$
\int_{0}^{T} \int_{\mathbb{R}^{N}}\left|u_{\varepsilon}(t, x)-u\left(t, x-\frac{b^{*} t}{\varepsilon}\right)\right|^{2} d x d t \underset{\varepsilon \rightarrow 0}{\longrightarrow} 0
$$

where $b^{*}$ is the homogenized velocity defined by

$$
b^{*}=\frac{\int_{Y} b(y) d y}{\rho^{*}} \quad \text { and } \quad \rho^{*}=\int_{Y} \rho(y) d y,
$$

while $u$ is the unique solution of the homogenized problem

$$
\begin{cases}\rho^{*} \frac{\partial u}{\partial t}-\operatorname{div}\left(A^{*} \nabla u\right)=0 & \text { in }(0, T) \times \mathbb{R}^{N}, \\ u(0, x)=u^{0}(x) & \text { in } \mathbb{R}^{N},\end{cases}
$$


with $A^{*}$ the homogenized diffusion tensor defined by its entries

$$
A_{i, j}^{*}=\int_{Y}\left(\rho(y) b_{i}^{*}-b_{i}(y)\right) w_{j} d y+\int_{Y} A(y)\left(\nabla_{y} w_{j}+e_{j}\right) \cdot e_{i} d y
$$

or, equivalently,

$$
A_{i, j}^{*}=\int_{Y} A(y)\left(\nabla_{y} w_{i}+e_{i}\right) \cdot\left(\nabla_{y} w_{j}+e_{j}\right) d y
$$

and $w_{i}$ the solution of the cell problem (2.12).

Another interpretation of Theorem 1 is that the solution $u_{\varepsilon}$ of (1.3) is approximately given by

$$
u_{\varepsilon}(t, x) \approx \tilde{u}_{\varepsilon}(t, x)
$$

where $\tilde{u}_{\varepsilon}$ verifies

$$
\tilde{u}_{\varepsilon}(t, x)=u\left(t, x-\frac{b^{*} t}{\varepsilon}\right)
$$

and is the solution of a modified homogenized problem

$$
\begin{cases}\rho^{*} \frac{\partial \tilde{u}_{\varepsilon}}{\partial t}+\frac{1}{\varepsilon} b^{*} \cdot \nabla \tilde{u}_{\varepsilon}-\operatorname{div}\left(A^{*} \nabla \tilde{u}_{\varepsilon}\right)=0 & \text { in }(0, T) \times \mathbb{R}^{N}, \\ \tilde{u}_{\varepsilon}(0, x)=u^{0}(x) & \text { in } \mathbb{R}^{N} .\end{cases}
$$

The interest of the comparison with $\tilde{u}_{\varepsilon}$ is that the frame of reference is the same for $u_{\varepsilon}$ and $\tilde{u}_{\varepsilon}$ and that the convective term is explicit in (2.6). Note that $\tilde{u}_{\varepsilon}(t, x)$ is not an oscillating function although it still depends on $\varepsilon$ because of the large convective term in (2.6).

REMARK 1. It is clear from the statement of Theorem 1 that, in the general case when $b^{*} \neq 0$, such a result cannot hold for a bounded domain. Indeed, for a bounded domain the leading asymptotic term

$u\left(t, x-\frac{b^{*} t}{\varepsilon}\right)$ escapes from the domain. In other words, the imposed boundary conditions will play a crucial role and change the asymptotic behavior (see [5] for more details). This explains why we work in the full space $\mathbb{R}^{N}$ instead.

\subsection{Asymptotic expansion with drift.}

A formal proof of Theorem 1 can be deduced from the method of two-scale asymptotic expansion with drift that we briefly recall. As in [1], [4], [6], [14], [22], [26], we assume that the solution $u_{\varepsilon}$ can be expressed by means of the following series:

$$
u_{\varepsilon}(t, x)=\sum_{i=0}^{+\infty} \varepsilon^{i} u_{i}\left(t, x-\frac{b^{*} t}{\varepsilon}, \frac{x}{\varepsilon}\right)
$$

where each function $u_{i}(t, x, y)$ is $Y$-periodic with respect to $y$ and $b^{*}$ is a constant vector which represents the homogenized velocity and is an unknown that will be determined later. We insert this expansion into (1.3). The identification of the terms corresponding to each power of $\varepsilon$ leads to the following set of equations:

$$
\begin{gathered}
b(y) \cdot \nabla_{y} u_{0}-\operatorname{div}_{y}\left(A(y) \nabla_{y} u_{0}\right)=0, \\
-\rho(y) b^{*} \cdot \nabla_{x} u_{0}+b(y) \cdot\left(\nabla_{x} u_{0}+\nabla_{y} u_{1}\right)-\operatorname{div}_{y}\left(A(y)\left(\nabla_{x} u_{0}+\nabla_{y} u_{1}\right)\right)=\operatorname{div}_{x}\left(A(y) \nabla_{y} u_{0}\right),
\end{gathered}
$$




$$
\begin{aligned}
b(y) \cdot \nabla_{y} u_{2}-\operatorname{div}_{y}\left(A(y) \nabla_{y} u_{2}\right)=-\rho(y) \partial_{t} u_{0}+\rho(y) b^{*} \cdot \nabla_{x} u_{1}-b(y) \cdot \nabla_{x} u_{1} \\
+\operatorname{div}_{y}\left(A(y) \nabla_{x} u_{1}\right)+\operatorname{div}_{x}\left(A(y)\left(\nabla_{y} u_{1}+\nabla_{x} u_{0}\right)\right),
\end{aligned}
$$

complemented by $Y$-periodic boundary conditions. From (2.8), we deduce that $u_{0}$ does not depend on the variable $y \in Y$ so that $u_{0}(t, x, y)=u(t, x)$ for any $y \in Y$. Then, $(2.9)$ can be rewritten as

$$
-\rho(y) b^{*} \cdot \nabla_{x} u_{0}+b(y) \cdot\left(\nabla_{x} u_{0}+\nabla_{y} u_{1}\right)-\operatorname{div}_{y}\left(A(y)\left(\nabla_{x} u_{0}+\nabla_{y} u_{1}\right)\right)=0 .
$$

From the compatibility (or solvability) condition of (2.11), we deduce that the homogenized velocity $b^{*}$ must be given by formula (2.1). Morevoer, for each $i=1, \ldots, N$, we introduce the function $w_{i}$, solution to the cell problem

$$
b(y) \cdot\left(\nabla_{y} w_{i}+e_{i}\right)-\operatorname{div}_{y}\left(A(y)\left(\nabla_{y} w_{i}+e_{i}\right)\right)=\rho(y) b^{*} \cdot e_{i}, \text { in } Y .
$$

The Fredholm alternative ensures the existence of a solution $w_{i} \in H_{\#}^{1}(Y)$ to this equation. Moreover, under the hypotheses 1 , we also have $w_{i} \in W_{\#}^{1, \infty}(Y)$. By linearity of $(2.11), u_{1}$ can be computed, up to an additive function of $x$, as

$$
u_{1}\left(t, x-\frac{b^{*} t}{\varepsilon}, y\right)=\sum_{i=1}^{N} \frac{\partial u}{\partial x_{i}}\left(t, x-\frac{b^{*} t}{\varepsilon}\right) w_{i}(y)
$$

Eventually, the compatibility condition of (2.10) yields the homogenized problem (2.2) for $u$. This is thus a formal proof of Theorem 1. A rigorous proof is obtained by using the notion of two-scale convergence with drift introduced in [22].

\subsection{A priori error estimate.}

Theorem 1 states that $u$ is a fair approximation of $u_{\varepsilon}$ with respect to the $L^{2}$ norm. However, it is not sufficient for higher order approximations and it was improved in [3] and [24] by using the corrector term $u_{1}$ as follows.

THEOREM 2. Let $u_{\varepsilon}$ be the sequence of solutions to (1.3), $u$ be the solution of the homogenized problem (2.2) and $u_{1}$ be given by (2.13). Assuming that Hypotheses 1 hold, then

$$
\left\|u_{\varepsilon}(t, x)-u\left(t, x-\frac{b^{*} t}{\varepsilon}\right)-\varepsilon u_{1}\left(t, x-\frac{b^{*} t}{\varepsilon}, \frac{x}{\varepsilon}\right)\right\|_{L^{2}\left((0, T), H^{1}\left(\mathbb{R}^{N}\right)\right)} \leqslant C \varepsilon,
$$

where $C>0$ depends on the final time $T$ but not on $\varepsilon$.

Inequality (2.14) allows us to justify the approximation

$$
u_{\varepsilon}(t, x) \approx u\left(t, x-\frac{b^{*} t}{\varepsilon}\right)+\varepsilon \sum_{i=1}^{N} \frac{\partial u}{\partial x_{i}}\left(t, x-\frac{b^{*} t}{\varepsilon}\right) w_{i}\left(\frac{x}{\varepsilon}\right)
$$

which will be the starting point of our new multiscale method.

The proof Theorem 2 is a consequence of the following technical lemma (for details, we refer to [3] and [24]).

Lemma 1. Under the same assumptions as Theorem 2, we introduce the remainder

$$
r_{\varepsilon}(t, x)=\varepsilon^{-1}\left(u_{\varepsilon}(t, x)-u_{0}\left(t, x-\frac{b^{*} t}{\varepsilon}\right)-\varepsilon u_{1}\left(t, x-\frac{b^{*} t}{\varepsilon}, \frac{x}{\varepsilon}\right)\right) .
$$

Then

$$
\left\|r_{\varepsilon}\right\|_{L^{\infty}\left((0, T), L^{2}\left(\mathbb{R}^{N}\right)\right)} \leqslant C \quad \text { and } \quad\left\|\nabla r_{\varepsilon}\right\|_{L^{2}\left((0, T) \times \mathbb{R}^{N}\right)^{N}} \leqslant C,
$$

where $C>0$ depends on the final time $T$ but not on $\varepsilon$. 


\section{A new multiscale finite element method.}

In this section, we recall the definition of our multiscale finite element method as introduced in [3] and [24]. Since it is not possible to discretize the full space $\mathbb{R}^{N}$, we replace it by a rectangular domain $\Omega$, complemented with periodic boundary conditions on $\partial \Omega$. The previous homogenization results, Theorems 1 and 2 , obviously extend to this simpler setting.

To enforce that the domain $\Omega$ is always the union of an entire number of periodicity cells we make the following additional assumption. We also replace point 6 of Hypotheses 1.

HYPOTHESES 2.

1. The sequence of real numbers $\varepsilon$ converging towards 0 is such that for each $\varepsilon$, there exist integers $\left(n_{i}^{\varepsilon}, m_{i}^{\varepsilon}\right)_{1 \leqslant i \leqslant N}$ such that

$$
\Omega=\prod_{i=1}^{N}\left(n_{i}^{\varepsilon} \varepsilon, m_{i}^{\varepsilon} \varepsilon\right), \quad n_{i}^{\varepsilon}, m_{i}^{\varepsilon} \in \mathbb{N} \text { with } n_{i}^{\varepsilon}<m_{i}^{\varepsilon} .
$$

2. $u^{0} \in W_{\#}^{k+3, \infty}(\Omega)$ where $k \in \mathbb{N} \backslash\{0\}$ is the order of the finite element method to be defined.

In other words, we replace problem (1.3) by

$$
\begin{cases}\rho^{\varepsilon}(x) \frac{\partial u_{\varepsilon}}{\partial t}+\frac{1}{\varepsilon} b^{\varepsilon}(x) \cdot \nabla u_{\varepsilon}-\operatorname{div}\left(A^{\varepsilon}(x) \nabla u_{\varepsilon}\right)=0 & \text { in }(0, T) \times \Omega, \\ x \rightarrow u_{\varepsilon}(t, x) \text { is } \Omega \text {-periodic } & \text { in }(0, T), \\ u_{\varepsilon}(0, x)=u^{0}(x) & \text { in } \Omega .\end{cases}
$$

\subsection{Idea of the method.}

As suggested in [19] and following an idea of L. Tartar [29], we introduce oscillating test functions

$$
\widehat{w}_{i}^{\varepsilon}=x_{i}+\varepsilon w_{i}\left(\frac{x}{\varepsilon}\right) \text {, }
$$

each $w_{i}\left(\frac{x}{\varepsilon}\right)$ being the solution of $(2.12)$. With this definition, we have

$$
\nabla \widehat{w}_{i}^{\varepsilon}(x)=e_{i}+\left(\nabla_{y} w_{i}\right)\left(\frac{x}{\varepsilon}\right) .
$$

Since $\operatorname{div}_{y}=\varepsilon \operatorname{div},(2.12)$ becomes

$$
\frac{1}{\varepsilon} b\left(\frac{x}{\varepsilon}\right) \cdot \nabla \widehat{w}_{i}^{\varepsilon}-\operatorname{div}\left(A\left(\frac{x}{\varepsilon}\right) \nabla \widehat{w}_{i}^{\varepsilon}\right)=\frac{1}{\varepsilon} \rho\left(\frac{x}{\varepsilon}\right) b^{*} \cdot e_{i} \quad \text { in } \varepsilon Y,
$$

where each function $\widehat{w}_{i}^{\varepsilon}$ is $\varepsilon$-periodic. Using the approximation (2.15), $u_{\varepsilon}$ satisfies

$$
u_{\varepsilon}(t, x) \approx u\left(t, x-\frac{b^{*} t}{\varepsilon}\right)+\sum_{i=1}^{N}\left(\widehat{w}_{i}^{\varepsilon}(x)-x_{i}\right) \frac{\partial u}{\partial x_{i}}\left(t, x-\frac{b^{*} t}{\varepsilon}\right) .
$$

Here, as in [2], it is important to notice that the right hand side of this approximation is a first-order Taylor expansion with respect to the space variable. Thus, equivalently, we have:

$$
u_{\varepsilon}(t, x) \approx u\left(t, \widehat{w}^{\varepsilon}(x)-\frac{b^{*} t}{\varepsilon}\right) .
$$

Introducing $\tilde{u}_{\varepsilon}$ defined in (2.5), we have

$$
u\left(t, \widehat{w}^{\varepsilon}(x)-\frac{b^{*} t}{\varepsilon}\right)=\tilde{u}_{\varepsilon}\left(t, \widehat{w}^{\varepsilon}(x)\right)
$$


and the previous approximation can be rewritten as:

$$
u_{\varepsilon}(t, x) \approx \tilde{u}_{\varepsilon}(t, \cdot) \circ \widehat{w}^{\varepsilon}(x) .
$$

The multiscale method presented in this paper is based on this approximation and a set of multiscale basis functions is built following this idea of composition. This change of variable, called harmonic coordinates, was first introduced by S. Kozlov in [21] and applied to define a multiscale method in [2] and [25].

\subsection{Coarse mesh and weak formulation.}

Let $\mathcal{K}_{H}$ be a family of meshes of resolution $H$ with $\bar{\Omega}=\overline{\bigcup_{K \in \mathcal{K}_{H}} K}$. A mesh $\mathcal{K}_{H}$ will be referred to as the coarse mesh. For each cell $K \in \mathcal{K}_{H}$, let us define:

- the diameter $H_{K}$ which is the length of the longest edge of $K$,

- the roundness $\rho_{K}$, the diameter of the inscribed ball in $K$,

- the excentricity $\sigma_{K}=\frac{H_{K}}{\rho_{K}}$ which measures the non-degeneracy of $K$.

Each mesh $\mathcal{K}_{H}$ is defined so that $H=\max _{K \in \mathcal{K}_{H}} H_{K}$. Here are recalled several definitions which will be used afterward.

Definition 1. A mesh $\mathcal{K}_{H}$ is conformal (in the finite element theory) if every face of an element $K \in \mathcal{K}_{H}$ is either part of the boundary $\partial \Omega$, or the face of another element $K^{\prime}$.

Definition 2. The family of meshes $\left(\mathcal{K}_{H}\right)_{H}$ is regular if and only if, there exists a constant $C_{r}$ such that

$$
\sigma_{K} \leqslant C_{r}
$$

for each cell $K \in \mathcal{K}_{H}$ and for all $H>0$.

Definition 3. The family of meshes $\left(\mathcal{K}_{H}\right)_{H}$ is quasi-uniform if and only if, there exists a constant $C>0$ such that

$$
\forall H>0, \forall K \in \mathcal{K}_{H}, \quad C H \leqslant \rho_{K} \leqslant H_{K} \leqslant H .
$$

In the following, we make the following assumption.

Hypotheses 3. The family of meshes $\left(\mathcal{K}_{H}\right)_{H}$ is conformal, regular and quasi-uniform.

We build a multiscale finite element method that can be applied to non-periodic cases. Therefore, in each coarse cell $K \in \mathcal{K}_{H}$, we define functions which are equivalent to the functions $\widehat{w}_{i}^{\varepsilon}$ in a non-periodic case. As a result, restating the definition (3.2) of $\widehat{w}_{i}^{\varepsilon}$, we define the functions $\widetilde{w}_{i}^{\varepsilon, K}$ as the solutions of

$$
\left\{\begin{aligned}
\frac{1}{\varepsilon} b^{\varepsilon}(x) \cdot \nabla \widetilde{w}_{i}^{\varepsilon, K}-\operatorname{div}\left(A^{\varepsilon}(x) \nabla \widetilde{w}_{i}^{\varepsilon, K}\right) & =\frac{1}{\varepsilon} \rho^{\varepsilon}(x) b_{K}^{*} \cdot e_{i} \text { in } K, \\
\widetilde{w}_{i}^{\varepsilon, K} & =\quad x_{i} \quad \text { on } \partial K,
\end{aligned}\right.
$$

where

$$
b_{K}^{*}=\frac{\int_{K} b^{\varepsilon}(x) d x}{\int_{K} \rho^{\varepsilon}(x) d x}
$$

In practice, (3.4) is solved, in each cell $K$, using a finite element method on a local fine mesh of resolution $h \ll H$. A function $\widetilde{w}_{i}^{\varepsilon, H}$ is then defined on $\Omega$ gathering all functions $\widetilde{w}_{i}^{\varepsilon, K}$ on each cell $K$. Given the regularity of the functions $b^{\varepsilon}, A^{\varepsilon}$ and $\rho^{\varepsilon}$, each function $\widetilde{w}_{i}^{\varepsilon, H}$ belongs to $W^{1, \infty}(\Omega)$. Finally, we define the vector-valued function $\widetilde{w}^{\varepsilon, H}=\left(\widetilde{w}_{i}^{\varepsilon, H}\right)_{i=1, \ldots, N}$. 


\subsection{Definition of the multiscale finite element space.}

Let $V_{H}$ be a linear subspace of $H_{\#}^{1}(\Omega)$ (the subspace of $H^{1}(\Omega)$ made of $\Omega$-periodic functions) associated to the coarse mesh $\mathcal{K}_{H}, D_{H}$ the dimension of this space, i.e., the number of degrees of freedom. In what follows, the subspace $V_{H}$ is a $\mathbb{P}_{k}$ Lagrange finite element space and $\left(\Phi_{l}^{H}\right)_{l \in \mathcal{N}_{\mathbb{P}_{k}, H}}$ is a basis of $V_{H}$. A new space $V_{\varepsilon, H}$ is then defined: it is spanned by the multiscale basis functions:

$$
\Phi_{l}^{\varepsilon, H}=\Phi_{l}^{H} \circ \widetilde{w}^{\varepsilon, H}, \quad l=1, \ldots, D_{H} .
$$

REMARK 2. Since for all $x \in \partial \Omega, \quad \widetilde{w}^{\varepsilon, H}(x)=x$, for any $\Omega$-periodic function $f, f \circ \widetilde{w}^{\varepsilon, H}$ is also $\Omega$-periodic.

Moreover, recalling that $\widehat{w}_{i}^{\varepsilon}(x)=x_{i}+\varepsilon w_{i}\left(\frac{x}{\varepsilon}\right)$ then , using Hypothesis 2.1, $\widehat{w}^{\varepsilon}$ is equal to the identity function to which an $\Omega$-periodic function is added. Therefore, for any $\Omega$-periodic function $f, f \circ \widehat{w}^{\varepsilon}$ is also $\Omega$-periodic. This remark proves that $V_{\varepsilon, H}$ is indeed a subspace of $H_{\#}^{1}(\Omega)$. Accordingly, we introduce the interpolation operator $\pi_{\varepsilon, H}$ associated to $V_{\varepsilon, H}$ :

$$
\pi_{\varepsilon, H} v(x)=\sum_{l \in \mathcal{N}_{\mathbb{P}_{k}, H}} v(l) \Phi_{l}^{\varepsilon, H}(x)=\sum_{l \in \mathcal{N}_{\mathbb{P}_{k}, H}} v(l) \Phi_{l}^{H} \circ \widetilde{w}^{\varepsilon, H}(x)=\left(\pi_{H} v\right) \circ \widetilde{w}^{\varepsilon, H}(x)
$$

where $\pi_{H}$ is the interpolation operator associated to $V_{H}$ :

$$
\pi_{H} v(x)=\sum_{l \in \mathcal{N}_{\mathbb{P}_{k}, H}} v(l) \Phi_{l}^{H}(x) .
$$

We seek a numerical approximation $u_{\varepsilon, H}$ in $V_{\varepsilon, H}$ of the exact solution $u_{\varepsilon}$. We compute it by means of the variational formulation: find $u_{\varepsilon, H} \in \mathcal{C}^{\infty}\left((0, T), V_{\varepsilon, H}\right)$ such that $u_{\varepsilon, H}(0, x)=\pi_{\varepsilon, H} u^{0}(x)$ and, for any $v_{\varepsilon, H} \in V_{\varepsilon, H}$,

$$
\left(D_{t} u_{\varepsilon, H}, v_{\varepsilon, H}\right)_{L^{2}(\Omega)}+a\left(u_{\varepsilon, H}, v_{\varepsilon, H}\right)=0,
$$

where $D_{t}$ is the convective operator $D_{t}=\rho^{\varepsilon} \frac{\partial}{\partial t}+\frac{1}{\varepsilon} b^{\varepsilon} \cdot \nabla$ and $a(u, v)=\int_{\Omega} A^{\varepsilon} \nabla u \cdot \nabla v d x$. In the rest of this article, we introduce simplifying notations:

$$
\partial_{t}=\frac{\partial}{\partial t} \quad \text { and } \quad \partial_{x_{i}}=\frac{\partial}{\partial x_{i}} .
$$

4. A priori error estimate of the multiscale finite element method.

This section is devoted to the main result of the present paper, that is an estimate of the error between the numerical solution obtained with our multiscale finite element method and the exact solution of problem (3.1).

TheOREM 3. There exists a constant $C$ which does not depend on $\varepsilon$ or $H$ such that

$$
\left\|u_{\varepsilon}-u_{\varepsilon, H}\right\|_{\Omega_{T}} \leqslant C\left(H^{k}+\frac{\left|b^{*}\right|}{\varepsilon}\left(H^{k+1}+\varepsilon\right)+\sqrt{\frac{\varepsilon}{H}}\right),
$$

where

$$
\|u\|_{\Omega_{T}}^{2}=\|u\|_{L^{\infty}\left((0, T), L^{2}(\Omega)\right)}^{2}+|u|_{L^{2}\left((0, T), H^{1}(\Omega)\right)}^{2} .
$$

We recall that $\varepsilon$ represents the size of the heterogeneities, $H$ the size of the coarse mesh and $k \geqslant 1$ is the order of the finite element method defined on the coarse mesh on which the multiscale method is based.

REMARK 3. The presence of the term $\frac{\left|b^{*}\right|}{\varepsilon}$ in the error estimate is rather inconvenient. Indeed, due to this factor, the upper bound in (4.1) does not tend to zero when $\varepsilon$ and $H$ get smaller. However, the multiscale 
method presented here does not treat specifically the convection term in the equation since it is based on a classical $\mathbb{P}_{k}$ Lagrange finite element method. In other words, the numerical scheme is centered and no upwinding is applied. As a result, we obtain this nasty term $\frac{\left|b^{*}\right|}{\varepsilon}$. Of course, we could always remember that the large convective term is coming from an adimensionalization process (as described in the introduction) and claim that, after all, the homogenized velocity $\frac{\left|b^{*}\right|}{\varepsilon}$ is of order 1 in the original physical variables. A better answer would be to improve our multiscale finite element method by using a better coarse discretization method like a SUPG method [10] or a Galerkin characteristic method ([9] or [27]). Unfortunately, we are not able to prove a better error estimate in these cases.

Proof. In order to prove this theorem, we will begin in Section 4.1 with a characterization of the error obtained when solving (3.1) in an approximation space (Lemma 2). This lemma is in fact an adaptation of Céa's lemma [12] for a transport equation. It is then applied with a specific test function:

$$
v_{\varepsilon, H}=\pi_{\varepsilon, H} \tilde{u}_{\varepsilon},
$$

where $\tilde{u}_{\varepsilon}(t, x)=u\left(t, x-\frac{b^{*} t}{\varepsilon}\right)$, and $u$ is the solution of the homogenized problem (2.2). This gives the following inequality:

$$
\left\|u_{\varepsilon}-u_{\varepsilon, H}\right\|_{\Omega_{T}} \leqslant C\left(X_{1}+X_{2}+X_{3}+X_{4}+X_{5}\right),
$$

where

$$
\begin{aligned}
X_{1} & =\left|u_{\varepsilon}-\pi_{\varepsilon, H} \tilde{u}_{\varepsilon}\right|_{L^{2}\left((0, T), H^{1}(\Omega)\right)}, \\
X_{2} & =\left\|u_{\varepsilon}-\pi_{\varepsilon, H} \tilde{u}_{\varepsilon}\right\|_{L^{\infty}\left((0, T), L^{2}(\Omega)\right)}, \\
X_{3} & =\left\|D_{t}\left(u_{\varepsilon}-\pi_{\varepsilon, H} \tilde{u}_{\varepsilon}\right)\right\|_{L^{2}\left((0, T), \dot{H}_{\#}^{-1}(\Omega)\right)}, \\
X_{4} & =\left\|\int_{\Omega} D_{t}\left(u_{\varepsilon}-\pi_{\varepsilon, H} \tilde{u}_{\varepsilon}\right)\right\|_{L^{2}((0, T))}, \\
\text { and } \quad X_{5} & =\left\|\left(u_{\varepsilon, H}-\pi_{\varepsilon, H} \tilde{u}_{\varepsilon}\right)(0, \cdot)\right\|_{L^{2}(\Omega)} .
\end{aligned}
$$

The term $X_{1}$ is bounded in Section 4.3, $X_{2}$ in Section 4.4, $X_{3}$ and $X_{4}$ in Section 4.5 and $X_{5}$ is bounded in Section 4.2 .

Adding inequalities (4.15), (4.36), (4.38) and (4.14) into (4.2) leads to the desired result (4.1).

4.1. The approximation error.

Let us define the subspace $\dot{H}_{\#}^{1}(\Omega)$ of $H_{\#}^{1}(\Omega)$ by

$$
\dot{H}_{\#}^{1}(\Omega)=\left\{\varphi \in H_{\#}^{1}(\Omega) \mid \int_{\Omega} \varphi=0\right\} .
$$

Its dual space is then denoted by $\dot{H}_{\#}^{-1}(\Omega)$ and the corresponding norm verifies

$$
\|u\|_{\dot{H}_{\#}^{-1}(\Omega)}=\max _{\varphi \in \dot{H}_{\#}^{1}(\Omega) \backslash\{0\}} \frac{\int_{\Omega} u \varphi}{\|\nabla \varphi\|_{L^{2}(\Omega)^{N}}} .
$$

Lemma 2. There exists a constant $C>0$ which does not depend on $\varepsilon$ such that

$$
\begin{aligned}
\left\|u_{\varepsilon}-u_{\varepsilon, H}\right\|_{\Omega_{T}} \leqslant C \inf _{v_{\varepsilon, H} \in \mathcal{C}^{\infty}\left((0, T), V_{\varepsilon, H}\right)}\left(\left\|u_{\varepsilon}-v_{\varepsilon, H}\right\|_{\Omega_{T}}+\left\|D_{t}\left(u_{\varepsilon}-v_{\varepsilon, H}\right)\right\|_{L^{2}\left((0, T), \dot{H}_{\#}^{-1}(\Omega)\right)}\right. \\
\left.+\left\|\int_{\Omega} D_{t}\left(u_{\varepsilon}-v_{\varepsilon, H}\right)\right\|_{L^{2}((0, T))}+\left\|\left(u_{\varepsilon, H}-v_{\varepsilon, H}\right)(0, \cdot)\right\|_{L^{2}(\Omega)}\right),
\end{aligned}
$$


where $D_{t}=\rho^{\varepsilon} \partial_{t}+\frac{1}{\varepsilon} b^{\varepsilon} \cdot \nabla$ and $\|\cdot\|_{\Omega_{T}}$ is defined in Theorem 3 .

The proof of Lemma 2 is based on the article [30] which is concerned with an equivalent inequality in the case of homogeneous Dirichlet boundary conditions. It also relies on the following lemma.

LEMma 3. There exists a constant $C>0$ such that

$$
\forall u \in L_{\#}^{2}(\Omega), \forall v \in H_{\#}^{1}(\Omega), \quad\left|\int_{\Omega} u v\right| \leqslant\|u\|_{\dot{H}_{\#}^{-1}(\Omega)}\|\nabla v\|_{L^{2}(\Omega)^{N}}+C\left|\int_{\Omega} u\right|\|v\|_{L^{2}(\Omega)} .
$$

Proof. The proof is here quite straightforward:

$$
\begin{aligned}
\left|\int_{\Omega} u v\right| & =\left|\int_{\Omega} u\left(v-\frac{1}{|\Omega|} \int_{\Omega} v\right)+\frac{1}{|\Omega|} \int_{\Omega} u \int_{\Omega} v\right| \\
& \leqslant\|u\|_{\dot{H}_{\#}^{-1}(\Omega)}\|\nabla v\|_{L^{2}(\Omega)^{N}}+\frac{1}{|\Omega|}\left|\int_{\Omega} u\right|\left|\int_{\Omega} v\right| \\
& \leqslant\|u\|_{\dot{H}_{\#}^{-1}(\Omega)}\|\nabla v\|_{L^{2}(\Omega)^{N}}+|\Omega|^{-\frac{1}{2}}\left|\int_{\Omega} u\right|\|v\|_{L^{2}(\Omega)}
\end{aligned}
$$

applying Cauchy-Schwarz inequality.

Proof of Lemma 2. By using Poincaré's inequality, it can be shown that $L_{\#}^{2}(\Omega) \subset \dot{H}_{\#}^{-1}(\Omega)$. Let $w_{\varepsilon, H} \in$ $\mathcal{C}^{\infty}\left((0, T), V_{\varepsilon, H}\right)$. Using the fact that $u_{\varepsilon}$ verifies $(1.3)$, for all $v_{\varepsilon, H} \in \mathcal{C}^{\infty}\left((0, T), V_{\varepsilon, H}\right)$

$$
\left(D_{t} w_{\varepsilon, H}, v_{\varepsilon, H}\right)_{L^{2}(\Omega)}+a\left(w_{\varepsilon, H}, v_{\varepsilon, H}\right)=\left(D_{t}\left(w_{\varepsilon, H}-u_{\varepsilon}\right), v_{\varepsilon, H}\right)_{L^{2}(\Omega)}+a\left(w_{\varepsilon, H}-u_{\varepsilon}, v_{\varepsilon, H}\right) .
$$

Then, subtracting (4.4) from (3.6) with $v_{\varepsilon, H}=u_{\varepsilon, H}-w_{\varepsilon, H}$, we get

$$
\begin{aligned}
\left(D_{t}\left(u_{\varepsilon, H}-w_{\varepsilon, H}\right), u_{\varepsilon, H}-w_{\varepsilon, H}\right)_{L^{2}(\Omega)}+a\left(u_{\varepsilon, H}-w_{\varepsilon, H}, u_{\varepsilon, H}-w_{\varepsilon, H}\right) & \\
= & \left(D_{t}\left(u_{\varepsilon}-w_{\varepsilon, H}\right), u_{\varepsilon, H}-w_{\varepsilon, H}\right)_{L^{2}(\Omega)}+a\left(u_{\varepsilon}-w_{\varepsilon, H}, u_{\varepsilon, H}-w_{\varepsilon, H}\right) .
\end{aligned}
$$

In the left-hand side of (4.5), the following properties are used:

$$
\left(D_{t}\left(u_{\varepsilon, H}-w_{\varepsilon, H}\right), u_{\varepsilon, H}-w_{\varepsilon, H}\right)_{L^{2}(\Omega)}=\frac{1}{2} \frac{d}{d t}\left\|\sqrt{\rho^{\varepsilon}}\left(u_{\varepsilon, H}-w_{\varepsilon, H}\right)\right\|_{L^{2}(\Omega)}^{2}
$$

because $\forall u \in H_{\#}^{1}(\Omega), \quad\left(b^{\varepsilon} \cdot \nabla u, u\right)_{L^{2}(\Omega)}=0$. And

$$
a\left(u_{\varepsilon, H}-w_{\varepsilon, H}, u_{\varepsilon, H}-w_{\varepsilon, H}\right) \geqslant C_{s t a}\left|u_{\varepsilon, H}-w_{\varepsilon, H}\right|_{H^{1}(\Omega)}^{2} .
$$

For the right-hand side, using Young's inequality

$$
\begin{aligned}
\left|a\left(u_{\varepsilon}-w_{\varepsilon, H}, u_{\varepsilon, H}-w_{\varepsilon, H}\right)\right| & \leqslant C_{b n d}\left|u_{\varepsilon}-w_{\varepsilon, H}\right|_{H^{1}(\Omega)}\left|u_{\varepsilon, H}-w_{\varepsilon, H}\right|_{H^{1}(\Omega)} \\
& \leqslant \frac{C_{s t a}}{4}\left|u_{\varepsilon, H}-w_{\varepsilon, H}\right|_{H^{1}(\Omega)}^{2}+\frac{C_{b n d}^{2}}{C_{s t a}}\left|u_{\varepsilon}-w_{\varepsilon, H}\right|_{H^{1}(\Omega)}^{2}
\end{aligned}
$$

and using Lemma 3

$$
\begin{aligned}
&\left|\left(D_{t}\left(w_{\varepsilon, H}-u_{\varepsilon}\right), u_{\varepsilon, H}-w_{\varepsilon, H}\right)_{L^{2}(\Omega)}\right| \leqslant\left\|D_{t}\left(u_{\varepsilon}-w_{\varepsilon, H}\right)\right\|_{\dot{H}_{\#}^{-1}(\Omega)}\left\|\nabla\left(u_{\varepsilon, H}-w_{\varepsilon, H}\right)\right\|_{L^{2}(\Omega)^{N}} \\
&+C\left|\int_{\Omega} D_{t}\left(u_{\varepsilon}-w_{\varepsilon, H}\right)\right|\left\|u_{\varepsilon, H}-w_{\varepsilon, H}\right\|_{L^{2}(\Omega)} \\
& \leqslant \frac{C_{\text {sta }}}{4}\left|u_{\varepsilon, H}-w_{\varepsilon, H}\right|_{H^{1}(\Omega)}^{2}+\frac{1}{C_{\text {sta }}}\left\|D_{t}\left(u_{\varepsilon}-w_{\varepsilon, H}\right)\right\|_{\dot{H}_{\#}^{-1}(\Omega)}^{2} \\
& \quad+\frac{\rho_{\text {min }}}{2}\left\|u_{\varepsilon, H}-w_{\varepsilon, H}\right\|_{L^{2}(\Omega)}^{2}+\frac{C^{2}}{2 \rho_{\text {min }}}\left|\int_{\Omega} D_{t}\left(u_{\varepsilon}-w_{\varepsilon, H}\right)\right|^{2} .
\end{aligned}
$$


Thus, we have,

$$
\begin{aligned}
& \frac{d}{d t}\left\|\sqrt{\rho^{\varepsilon}}\left(u_{\varepsilon, H}-w_{\varepsilon, H}\right)\right\|_{L^{2}(\Omega)}^{2} \leqslant \frac{2 C_{b n d}^{2}}{C_{\text {sta }}}\left|u_{\varepsilon}-w_{\varepsilon, H}\right|_{H^{1}(\Omega)}^{2} \\
&+\frac{2}{C_{\text {sta }}}\left\|D_{t}\left(u_{\varepsilon}-w_{\varepsilon, H}\right)\right\|_{\dot{H}_{\#}^{-1}(\Omega)}^{2}+\rho_{\min }\left\|u_{\varepsilon, H}-w_{\varepsilon, H}\right\|_{L^{2}(\Omega)}^{2} \\
& \quad+\frac{C^{2}}{\rho_{\text {min }}}\left|\int_{\Omega} D_{t}\left(u_{\varepsilon}-w_{\varepsilon, H}\right)\right|^{2}-C_{s t a}\left|u_{\varepsilon, H}-w_{\varepsilon, H}\right|_{H^{1}(\Omega)}^{2} .
\end{aligned}
$$

Using $\rho^{\varepsilon}(x) \geqslant \rho_{\text {min }}$, this leads to

$$
\begin{aligned}
\frac{d}{d t}\left\|\sqrt{\rho^{\varepsilon}}\left(u_{\varepsilon, H}-w_{\varepsilon, H}\right)\right\|_{L^{2}(\Omega)}^{2} \leqslant\left\|\sqrt{\rho^{\varepsilon}}\left(u_{\varepsilon, H}-w_{\varepsilon, H}\right)\right\|_{L^{2}(\Omega)}^{2} & \\
+C\left(\left|u_{\varepsilon}-w_{\varepsilon, H}\right|_{H^{1}(\Omega)}^{2}\right. & +\left\|D_{t}\left(u_{\varepsilon}-w_{\varepsilon, H}\right)\right\|_{\dot{H}_{\#}^{-1}(\Omega)}^{2} \\
& \left.+\left|\int_{\Omega} D_{t}\left(u_{\varepsilon}-w_{\varepsilon, H}\right)\right|^{2}\right)-C_{s t a}\left|u_{\varepsilon, H}-w_{\varepsilon, H}\right|_{H^{1}(\Omega)}^{2} .
\end{aligned}
$$

Then, applying Gronwall's inequality between 0 and $t \in(0, T)$ :

$$
\begin{aligned}
\left\|\sqrt{\rho^{\varepsilon}}\left(u_{\varepsilon, H}-w_{\varepsilon, H}\right)(t, \cdot)\right\|_{L^{2}(\Omega)}^{2} \leqslant \mathrm{e}^{t} \| & \sqrt{\rho^{\varepsilon}}\left(u_{\varepsilon, H}-w_{\varepsilon, H}\right)(0, \cdot) \|_{L^{2}(\Omega)}^{2} \\
+C \int_{0}^{t} \mathrm{e}^{t-s}\left(\left|u_{\varepsilon}-w_{\varepsilon, H}\right|_{H^{1}(\Omega)}^{2}+\left\|D_{t}\left(u_{\varepsilon}-w_{\varepsilon, H}\right)\right\|_{H_{\#}^{-1}(\Omega)}^{2}\right. & \\
& \left.+\left|\int_{\Omega} D_{t}\left(u_{\varepsilon}-w_{\varepsilon, H}\right)\right|^{2} d s\right)-C_{s t a} \int_{0}^{t} \mathrm{e}^{t-s}\left|u_{\varepsilon, H}-w_{\varepsilon, H}\right|_{H^{1}(\Omega)}^{2} d s .
\end{aligned}
$$

Since

$$
\forall s \in(0, t), \quad 1 \leqslant \mathrm{e}^{t-s} \leqslant \mathrm{e}^{t},
$$

we obtain

$$
\begin{aligned}
& \left\|\sqrt{\rho^{\varepsilon}}\left(u_{\varepsilon, H}-w_{\varepsilon, H}\right)(t, \cdot)\right\|_{L^{2}(\Omega)}^{2}+C_{s t a}\left|u_{\varepsilon, H}-w_{\varepsilon, H}\right|_{L^{2}\left((0, t), H^{1}(\Omega)\right)}^{2} \\
& \leqslant \mathrm{e}^{t}\left\|\sqrt{\rho^{\varepsilon}}\left(u_{\varepsilon, H}-w_{\varepsilon, H}\right)(0, \cdot)\right\|_{L^{2}(\Omega)}^{2} \\
& +C \mathrm{e}^{t}\left(\left|u_{\varepsilon}-w_{\varepsilon, H}\right|_{L^{2}\left((0, t), H^{1}(\Omega)\right)}^{2}+\left\|D_{t}\left(u_{\varepsilon}-w_{\varepsilon, H}\right)\right\|_{L^{2}\left((0, t), \dot{H}_{\#}^{-1}(\Omega)\right)}^{2}\right. \\
& \left.+\left\|\int_{\Omega} D_{t}\left(u_{\varepsilon}-w_{\varepsilon, H}\right)\right\|_{L^{2}(0, t)}^{2}\right) .
\end{aligned}
$$

Thus

$$
\begin{aligned}
& \left\|\sqrt{\rho^{\varepsilon}}\left(u_{\varepsilon, H}-w_{\varepsilon, H}\right)(t, \cdot)\right\|_{L^{2}(\Omega)}^{2} \leqslant \mathrm{e}^{T}\left\|\sqrt{\rho^{\varepsilon}}\left(u_{\varepsilon, H}-w_{\varepsilon, H}\right)(0, \cdot)\right\|_{L^{2}(\Omega)}^{2} \\
& +C \mathrm{e}^{T}\left(\left|u_{\varepsilon}-w_{\varepsilon, H}\right|_{L^{2}\left((0, T), H^{1}(\Omega)\right)}^{2}+\left\|D_{t}\left(u_{\varepsilon}-w_{\varepsilon, H}\right)\right\|_{L^{2}\left((0, T), \dot{H}_{\#}^{-1}(\Omega)\right)}^{2}\right. \\
& \left.\quad+\left\|\int_{\Omega} D_{t}\left(u_{\varepsilon}-w_{\varepsilon, H}\right)\right\|_{L^{2}(0, T)}^{2}\right) .
\end{aligned}
$$


This inequality is verified for every $t \in[0, T]$, so

$$
\begin{aligned}
\left\|\sqrt{\rho^{\varepsilon}}\left(u_{\varepsilon, H}-w_{\varepsilon, H}\right)\right\|_{L^{\infty}\left((0, T), L^{2}(\Omega)\right)}^{2} & \leqslant C\left(\left\|\sqrt{\rho^{\varepsilon}}\left(u_{\varepsilon, H}-w_{\varepsilon, H}\right)(0, \cdot)\right\|_{L^{2}(\Omega)}^{2}\right. \\
& +\left|u_{\varepsilon}-w_{\varepsilon, H}\right|_{L^{2}\left((0, T), H^{1}(\Omega)\right)}^{2} \\
& \left.+\left\|D_{t}\left(u_{\varepsilon}-w_{\varepsilon, H}\right)\right\|_{L^{2}\left((0, T), \dot{H}_{\#}^{-1}(\Omega)\right)}^{2}+\left\|\int_{\Omega} D_{t}\left(u_{\varepsilon}-w_{\varepsilon, H}\right)\right\|_{L^{2}(0, T)}^{2}\right) .
\end{aligned}
$$

In the same way, using inequality (4.9),

$$
\begin{aligned}
C_{s t a}\left|u_{\varepsilon, H}-w_{\varepsilon, H}\right|_{L^{2}\left((0, t), H^{1}(\Omega)\right)}^{2} \leqslant \mathrm{e}^{t} \| & \sqrt{\rho^{\varepsilon}}\left(u_{\varepsilon, H}-w_{\varepsilon, H}\right)(0, \cdot) \|_{L^{2}(\Omega)}^{2}+C \mathrm{e}^{t}\left(\left|u_{\varepsilon}-w_{\varepsilon, H}\right|_{L^{2}\left((0, t), H^{1}(\Omega)\right)}^{2}\right. \\
& \left.+\left\|D_{t}\left(u_{\varepsilon}-w_{\varepsilon, H}\right)\right\|_{L^{2}\left((0, t), \dot{H}_{\#}^{-1}(\Omega)\right)}^{2}+\left\|\int_{\Omega} D_{t}\left(u_{\varepsilon}-w_{\varepsilon, H}\right)\right\|_{L^{2}(0, t)}^{2}\right)
\end{aligned}
$$

and choosing $t=T$,

$$
\begin{aligned}
\left|u_{\varepsilon, H}-w_{\varepsilon, H}\right|_{L^{2}\left((0, T), H^{1}(\Omega)\right)}^{2} \leqslant & C\left(\left\|\sqrt{\rho^{\varepsilon}}\left(u_{\varepsilon, H}-w_{\varepsilon, H}\right)(0, \cdot)\right\|_{L^{2}(\Omega)}^{2}+\left|u_{\varepsilon}-w_{\varepsilon, H}\right|_{L^{2}\left((0, T), H^{1}(\Omega)\right)}^{2}\right. \\
& \left.+\left\|D_{t}\left(u_{\varepsilon}-w_{\varepsilon, H}\right)\right\|_{L^{2}\left((0, T), \dot{H}_{\#}^{-1}(\Omega)\right)}^{2}+\left\|\int_{\Omega} D_{t}\left(u_{\varepsilon}-w_{\varepsilon, H}\right)\right\|_{L^{2}(0, T)}^{2}\right) .
\end{aligned}
$$

Adding inequalities (4.10) and (4.11) leads to

$$
\begin{aligned}
& \left\|\sqrt{\rho^{\varepsilon}}\left(u_{\varepsilon, H}-w_{\varepsilon, H}\right)\right\|_{L^{\infty}\left((0, T), L^{2}(\Omega)\right)}^{2}+\left|u_{\varepsilon, H}-w_{\varepsilon, H}\right|_{L^{2}\left((0, T), H^{1}(\Omega)\right)}^{2} \\
& \leqslant C\left(\left|u_{\varepsilon}-w_{\varepsilon, H}\right|_{L^{2}\left((0, T), H^{1}(\Omega)\right)}^{2}+\left\|D_{t}\left(u_{\varepsilon}-w_{\varepsilon, H}\right)\right\|_{L^{2}\left((0, T), \dot{H}_{\#}^{-1}(\Omega)\right)}^{2}\right. \\
& \left.\quad+\left\|\int_{\Omega} D_{t}\left(u_{\varepsilon}-w_{\varepsilon, H}\right)\right\|_{L^{2}(0, T)}^{2}+\left\|\sqrt{\rho^{\varepsilon}}\left(u_{\varepsilon, H}-w_{\varepsilon, H}\right)(0, \cdot)\right\|_{L^{2}(\Omega)}^{2}\right) .
\end{aligned}
$$

Since $\rho^{\varepsilon} \geqslant \rho_{\min }$ and $\rho^{\varepsilon} \leqslant \rho_{\max }$,

$$
\begin{aligned}
& \left\|u_{\varepsilon, H}-w_{\varepsilon, H}\right\|_{L^{\infty}\left((0, T), L^{2}(\Omega)\right)}^{2}+\left|u_{\varepsilon, H}-w_{\varepsilon, H}\right|_{L^{2}\left((0, T), H^{1}(\Omega)\right)}^{2} \\
& \leqslant C\left(\left|u_{\varepsilon}-w_{\varepsilon, H}\right|_{L^{2}\left((0, T), H^{1}(\Omega)\right)}^{2}+\left\|D_{t}\left(u_{\varepsilon}-w_{\varepsilon, H}\right)\right\|_{L^{2}\left((0, T), \dot{H}_{\#}^{-1}(\Omega)\right)}^{2}\right. \\
& \left.\quad+\left\|\int_{\Omega} D_{t}\left(u_{\varepsilon}-w_{\varepsilon, H}\right)\right\|_{L^{2}(0, T)}^{2}+\left\|\left(u_{\varepsilon, H}-w_{\varepsilon, H}\right)(0, \cdot)\right\|_{L^{2}(\Omega)}^{2}\right) .
\end{aligned}
$$

This implies

$$
\begin{aligned}
\left\|u_{\varepsilon}-u_{\varepsilon, H}\right\|_{\Omega_{T}} \leqslant C\left(\left\|u_{\varepsilon}-w_{\varepsilon, H}\right\|_{\Omega_{T}}+\| D_{t}(\right. & \left.u_{\varepsilon}-w_{\varepsilon, H}\right) \|_{L^{2}\left((0, T), \dot{H}_{\#}^{-1}(\Omega)\right)} \\
& \left.+\left\|\int_{\Omega} D_{t}\left(u_{\varepsilon}-w_{\varepsilon, H}\right)\right\|_{L^{2}(0, T)}+\left\|\left(u_{\varepsilon, H}-w_{\varepsilon, H}\right)(0, \cdot)\right\|_{L^{2}(\Omega)}\right) .
\end{aligned}
$$




\subsection{The initial error $X_{5}$.}

The initial error $X_{5}$ is yet to be bounded:

$$
X_{5}=\left\|\left(u_{\varepsilon, H}-\pi_{\varepsilon, H} \tilde{u}_{\varepsilon}\right)(0, \cdot)\right\|_{L^{2}(\Omega)} .
$$

The initial condition of $u_{\varepsilon, H}$ is

$$
\forall x \in \Omega, \quad u_{\varepsilon, H}(0, x)=\pi_{\varepsilon, H} u^{0}(x) .
$$

The function $\tilde{u}_{\varepsilon}$ is defined by

$$
\tilde{u}_{\varepsilon}(t, x)=u\left(t, x-\frac{b^{*} t}{\varepsilon}\right)
$$

where $u$ is the solution of the cell problem (2.2) :

$$
\left\{\begin{array}{cl}
\rho^{*} \partial_{t} u-\operatorname{div}\left(A^{*} \nabla u\right) & =0 \quad \text { in } \mathbb{R}^{N} \times(0, T), \\
u(0, x) & =u^{0}(x) \text { in } \mathbb{R}^{N} .
\end{array}\right.
$$

Therefore

$$
\pi_{\varepsilon, H} \tilde{u}_{\varepsilon}(0, \cdot)=\pi_{\varepsilon, H} u^{0} .
$$

Hence

$$
\left\|\left(u_{\varepsilon, H}-\pi_{\varepsilon, H} \tilde{u}_{\varepsilon}\right)(0, \cdot)\right\|_{L^{2}(\Omega)}=0 .
$$

4.3. The gradient term $X_{1}$. Let us prove the following proposition.

Proposition 1. Let $u_{\varepsilon}$ be the solution of problem (3.1) and $\tilde{u}_{\varepsilon}$ defined by (2.5), then

$$
\left\|\nabla u_{\varepsilon}-\nabla \pi_{\varepsilon, H} \tilde{u}_{\varepsilon}\right\|_{L^{2}((0, T) \times \Omega)^{N}} \leqslant C\left(H^{k}+\sqrt{\frac{\varepsilon}{H}}\right),
$$

where $\pi_{\varepsilon, H}$ is the interpolation operator associated with $V_{\varepsilon, H}$ verifying (3.5).

Proof. First of all the norm is split into three terms:

$$
\left\|\nabla u_{\varepsilon}-\nabla \pi_{\varepsilon, H} \tilde{u}_{\varepsilon}\right\|_{L^{2}((0, T) \times \Omega)^{N}} \leqslant G_{1}+G_{2}+G_{3},
$$

where

$$
\begin{aligned}
G_{1} & =\left\|\nabla u_{\varepsilon}-\nabla\left(\tilde{u}_{\varepsilon}(t, \cdot) \circ \widehat{w}^{\varepsilon}\right)\right\|_{L^{2}((0, T) \times \Omega)^{N}}, \\
G_{2} & =\left\|\nabla\left(\left(\tilde{u}_{\varepsilon}(t, \cdot)-\pi_{H} \tilde{u}_{\varepsilon}(t, \cdot)\right) \circ \widehat{w}^{\varepsilon}\right)\right\|_{L^{2}((0, T) \times \Omega)^{N}}, \\
\text { and } \quad G_{3} & =\left\|\nabla\left(\pi_{H} \tilde{u}_{\varepsilon}(t, \cdot) \circ \widehat{w}^{\varepsilon}-\pi_{\varepsilon, H} \tilde{u}_{\varepsilon}\right)\right\|_{L^{2}((0, T) \times \Omega)^{N}},
\end{aligned}
$$

the function $\widehat{w}^{\varepsilon}$ is defined in each direction $i$ by

$$
\widehat{w}_{i}^{\varepsilon}(x)=x_{i}+\varepsilon w_{i}\left(\frac{x}{\varepsilon}\right),
$$

and the functions $w_{i}$ are the solutions of the cell problems (2.12). $G_{1}$ is a term of global homogenization which will be bounded by restating accurately the approximation (3.3) and it is bounded in Section 4.3.1. $G_{2}$ is a term of interpolation on the coarse mesh bounded in Section 4.3.2. $G_{3}$ is bounded in Section 4.3.3 by using homogenization results in each coarse cell.

The proof is obtained by collecting the results from Lemmata 4,5 and 7 that is to say by inserting inequalities (4.17), (4.23) and (4.27) into (4.16):

$$
\left\|\nabla u_{\varepsilon}-\nabla \pi_{\varepsilon, H} \tilde{u}_{\varepsilon}\right\|_{L^{2}((0, T) \times \Omega)^{N}} \leqslant C\left(\varepsilon+H^{k}+\sqrt{\frac{\varepsilon}{H}}\right) .
$$

Using the fact that $\varepsilon \leqslant C \sqrt{\frac{\varepsilon}{H}}$ because $\sqrt{\varepsilon H}$ is bounded leads to the inequality forecast by proposition 1 . 


\subsubsection{The global homogenization term $G_{1}$.}

Let us prove the following lemma.

Lemma 4. Assuming that the hypotheses of Proposition 1 are satisfied,

$$
\left\|\nabla u_{\varepsilon}-\nabla\left(\tilde{u}_{\varepsilon}(t, \cdot) \circ \widehat{w}^{\varepsilon}\right)\right\|_{L^{2}((0, T) \times \Omega)^{N}} \leqslant C \varepsilon .
$$

Proof. The problem is defined on the parallelepiped $\Omega$ and periodic boundary conditions are imposed, inequality (2.14), proved on the whole domain $\mathbb{R}^{N}$, still holds:

$$
\left\|u_{\varepsilon}(t, x)-u\left(t, x-\frac{b^{*} t}{\varepsilon}\right)-\varepsilon u_{1}\left(t, x-\frac{b^{*} t}{\varepsilon}, \frac{x}{\varepsilon}\right)\right\|_{L^{2}\left((0, T), H^{1}(\Omega)\right)} \leqslant C \varepsilon .
$$

First, the norm that has to be bounded is split into two parts, using Einstein summation convention:

$$
\begin{aligned}
\left\|\nabla u_{\varepsilon}-\nabla\left(\tilde{u}_{\varepsilon}(t, \cdot) \circ \widehat{w}^{\varepsilon}\right)\right\|_{L^{2}((0, T) \times \Omega)^{N}} \leqslant\left\|\nabla u_{\varepsilon}-\nabla \widehat{w}_{i}^{\varepsilon} \partial_{x_{i}} \tilde{u}_{\varepsilon}\right\|_{L^{2}((0, T) \times \Omega)^{N}} \\
+\left\|\nabla \widehat{w}_{i}^{\varepsilon} \partial_{x_{i}} \tilde{u}_{\varepsilon}-\nabla\left(\tilde{u}_{\varepsilon}(t, \cdot) \circ \widehat{w}^{\varepsilon}\right)\right\|_{L^{2}((0, T) \times \Omega)^{N}} .
\end{aligned}
$$

The first term is bounded thanks to inequality (4.18). Indeed

$$
\begin{aligned}
\nabla\left(u\left(t, x-\frac{b^{*} t}{\varepsilon}\right)+\varepsilon u_{1}\left(t, x-\frac{b^{*} t}{\varepsilon}, \frac{x}{\varepsilon}\right)\right) & =\nabla \tilde{u}_{\varepsilon}(t, x)+\varepsilon \nabla\left(w_{i}\left(\frac{x}{\varepsilon}\right) \frac{\partial u}{\partial x_{i}}\left(t, x-\frac{b^{*} t}{\varepsilon}\right)\right) \\
& =\left(e_{i}+\nabla_{y} w_{i}\left(\frac{x}{\varepsilon}\right)\right) \partial_{x_{i}} \tilde{u}_{\varepsilon}(t, x)+\varepsilon w_{i}\left(\frac{x}{\varepsilon}\right) \nabla \partial_{x_{i}} \tilde{u}_{\varepsilon} .
\end{aligned}
$$

And

$$
\nabla\left(u_{\varepsilon}(t, x)-u\left(t, x-\frac{b^{*} t}{\varepsilon}\right)-\varepsilon u_{1}\left(t, x-\frac{b^{*} t}{\varepsilon}, \frac{x}{\varepsilon}\right)\right)=\nabla u_{\varepsilon}-\nabla \widehat{w}_{i}^{\varepsilon} \partial_{x_{i}} \tilde{u}_{\varepsilon}-\varepsilon w_{i}\left(\frac{x}{\varepsilon}\right) \nabla \partial_{x_{i}} \tilde{u}_{\varepsilon} .
$$

Thus, restating inequality (4.18)

$$
\left\|\nabla u_{\varepsilon}-\nabla \widehat{w}_{i}^{\varepsilon} \partial_{x_{i}} \tilde{u}_{\varepsilon}\right\|_{L^{2}((0, T) \times \Omega)^{N}} \leqslant C \varepsilon+\varepsilon\left\|w_{i}\left(\frac{x}{\varepsilon}\right) \nabla \partial_{x_{i}} \tilde{u}_{\varepsilon}\right\|_{L^{2}((0, T) \times \Omega)^{N}} .
$$

Then, the fact that $u \in L^{\infty}\left((0, T), W^{k+2, \infty}(\Omega)\right)$ and $w \in L^{\infty}(Y)^{N}$ leads to

$$
\left\|\nabla u_{\varepsilon}-\nabla \widehat{w}_{i}^{\varepsilon} \partial_{x_{i}} \tilde{u}_{\varepsilon}\right\|_{L^{2}((0, T) \times \Omega)^{N}} \leqslant C \varepsilon .
$$

In order to bound the other term of (4.19), let us first rewrite it

$$
\nabla \widehat{w}_{i}^{\varepsilon} \partial_{x_{i}} \tilde{u}_{\varepsilon}-\nabla\left(\tilde{u}_{\varepsilon}(t, \cdot) \circ \widehat{w}^{\varepsilon}\right)=\nabla \widehat{w}_{i}^{\varepsilon}(x)\left(\partial_{x_{i}} \tilde{u}_{\varepsilon}(t, x)-\partial_{x_{i}} \tilde{u}_{\varepsilon}\left(t, \widehat{w}^{\varepsilon}(x)\right)\right) .
$$

Thus,

$$
\begin{aligned}
& \left\|\nabla \widehat{w}_{i}^{\varepsilon}(x)\left(\partial_{x_{i}} \tilde{u}_{\varepsilon}(t, x)-\partial_{x_{i}} \tilde{u}_{\varepsilon}\left(t, \widehat{w}^{\varepsilon}(x)\right)\right)\right\|_{L^{2}((0, T) \times \Omega)^{N}} \\
& \quad \leqslant\left\|I d+\nabla_{y} w\right\|_{L^{\infty}(Y)^{N \times N}}\left\|\nabla \tilde{u}_{\varepsilon}(t, x)-\nabla \tilde{u}_{\varepsilon}\left(t, \widehat{w}^{\varepsilon}(x)\right)\right\|_{L^{2}((0, T) \times \Omega)^{N}} .
\end{aligned}
$$

Writing the Taylor expansion of $\nabla \tilde{u}_{\varepsilon}$ gives:

$$
\nabla \tilde{u}_{\varepsilon}(t, \cdot) \circ \widehat{w}^{\varepsilon}(x)=\nabla \tilde{u}_{\varepsilon}(t, x)+\varepsilon \int_{0}^{1} w_{i}\left(\frac{x}{\varepsilon}\right) \nabla \partial_{x_{i}} \tilde{u}_{\varepsilon}\left(t, x+\varepsilon s w\left(\frac{x}{\varepsilon}\right)\right) d s .
$$


As a consequence

$$
\left\|\nabla \tilde{u}_{\varepsilon}(t, \cdot) \circ \widehat{w}^{\varepsilon}-\nabla \tilde{u}_{\varepsilon}\right\|_{L^{2}((0, T) \times \Omega)^{N}} \leqslant \varepsilon\left\|\tilde{u}_{\varepsilon}\right\|_{L^{2}\left((0, T), W^{2, \infty}(\Omega)\right)}\|w\|_{L^{\infty}(Y)^{N}} .
$$

Since $\tilde{u}_{\varepsilon}(t, x)=u\left(t, x-\frac{b^{*} t}{\varepsilon}\right)$ and periodic boundary conditions are imposed on $\Omega$,

$$
\left\|\tilde{u}_{\varepsilon}\right\|_{L^{2}\left((0, T), W^{2, \infty}(\Omega)\right)}=\|u\|_{L^{2}\left((0, T), W^{2, \infty}(\Omega)\right)} \leqslant \sqrt{T}\|u\|_{L^{\infty}\left((0, T), W^{2, \infty}(\Omega)\right)},
$$

using Cauchy-Schwarz inequality. So

$$
\left\|\nabla \tilde{u}_{\varepsilon}(t, \cdot) \circ \widehat{w}^{\varepsilon}-\nabla \tilde{u}_{\varepsilon}\right\|_{L^{2}((0, T) \times \Omega)^{N}} \leqslant C \varepsilon .
$$

Finally, injecting this inequality into (4.21) leads to

$$
\left\|\nabla \widehat{w}_{i}^{\varepsilon}(x)\left(\partial_{x_{i}} \tilde{u}_{\varepsilon}(t, x)-\partial_{x_{i}} \tilde{u}_{\varepsilon}\left(t, \widehat{w}^{\varepsilon}(x)\right)\right)\right\|_{L^{2}((0, T) \times \Omega)^{N}} \leqslant C \varepsilon .
$$

Then, adding inequalities (4.20) and (4.22) in (4.19) gives the desired result.

\subsubsection{The interpolation term $G_{2}$.}

Lemma 5. Under the same hypotheses as in Proposition 1,

$$
\left\|\nabla\left(\left(\tilde{u}_{\varepsilon}(t, \cdot)-\pi_{H} \tilde{u}_{\varepsilon}(t, \cdot)\right) \circ \widehat{w}^{\varepsilon}\right)\right\|_{L^{2}((0, T) \times \Omega)^{N}} \leqslant C H^{k}\|u\|_{L^{\infty}\left((0, T), W^{k+1, \infty}(\Omega)\right)},
$$

where $\pi_{H}$ is the interpolation operator on $V_{H}$.

Proof. First of all,

$$
\begin{aligned}
& \left\|\nabla\left(\left(\tilde{u}_{\varepsilon}(t, \cdot)-\pi_{H} \tilde{u}_{\varepsilon}(t, \cdot)\right) \circ \widehat{w}^{\varepsilon}\right)\right\|_{L^{2}((0, T) \times \Omega)^{N}} \\
& \quad=\left\|\nabla \widehat{w}_{i}^{\varepsilon} \partial_{x_{i}}\left(\tilde{u}_{\varepsilon}-\pi_{H} \tilde{u}_{\varepsilon}\right)(t, \cdot) \circ \widehat{w}^{\varepsilon}\right\|_{L^{2}((0, T) \times \Omega)^{N}} \\
& \quad \leqslant\left\|I d+\nabla_{y} w\right\|_{L^{\infty}(Y)^{N \times N}}\left\|\left(\nabla\left(\tilde{u}_{\varepsilon}-\pi_{H} \tilde{u}_{\varepsilon}\right)\right)(t, \cdot) \circ \widehat{w}^{\varepsilon}\right\|_{L^{2}((0, T) \times \Omega)^{N}},
\end{aligned}
$$

since the functions $w_{i}$ are in $W_{\#}^{1, \infty}(Y)$. The function $u$ belongs to the space

$$
L^{\infty}\left((0, T), W^{k+1, \infty}(\Omega)\right)
$$

so that

$$
\begin{aligned}
& \left\|\nabla\left(\left(\tilde{u}_{\varepsilon}(t, \cdot)-\pi_{H} \tilde{u}_{\varepsilon}(t, \cdot)\right) \circ \widehat{w}^{\varepsilon}\right)\right\|_{L^{2}((0, T) \times \Omega)^{N}} \\
& \quad \leqslant \sqrt{T|\Omega|}\left\|I d+\nabla_{y} w\right\|_{L^{\infty}(Y)^{N \times N}}\left\|\nabla\left(\tilde{u}_{\varepsilon}-\pi_{H} \tilde{u}_{\varepsilon}\right)\right\|_{L^{\infty}((0, T) \times \Omega)^{N}} .
\end{aligned}
$$

Then, using classical interpolation results (see [13]) and the fact that

$$
\left\|\tilde{u}_{\varepsilon}\right\|_{L^{\infty}\left((0, T), W^{k+1, \infty}(\Omega)\right)}=\|u\|_{L^{\infty}\left((0, T), W^{k+1, \infty}(\Omega)\right)}
$$

proves the lemma. $\square$

\subsubsection{The local homogenization term $G_{3}$.}

To achieve the proof of Proposition 1, a term still needs to be bounded:

$$
G_{3}=\left\|\nabla\left(\pi_{H} \tilde{u}_{\varepsilon}(t, \cdot) \circ \widehat{w}^{\varepsilon}-\pi_{\varepsilon, H} \tilde{u}_{\varepsilon}\right)\right\|_{L^{2}((0, T) \times \Omega)^{N}} .
$$

Let us first precise the error between $\widehat{w}^{\varepsilon}$ and $\widetilde{w}^{\varepsilon, K}$ using the following lemma. 
Lemma 6. There exists a constant $C$ which does not depend on $\varepsilon$ and $K$ such that

$$
\left|\widehat{w}^{\varepsilon}-\widetilde{w}^{\varepsilon, K}\right|_{H^{1}(K)^{N}} \leqslant C \sqrt{\varepsilon|\partial K|} .
$$

Moreover, there is a constant $C$ which also does not depend on $\varepsilon$ and $K$ such that

$$
\left\|\widehat{w}^{\varepsilon}-\widetilde{w}^{\varepsilon, K}\right\|_{L^{2}(K)^{N}} \leqslant C \varepsilon \sqrt{|K|}
$$

and

$$
\left\|\widehat{w}^{\varepsilon}-\widetilde{w}^{\varepsilon, K}\right\|_{L^{\infty}(K)^{N}} \leqslant\|w\|_{L^{\infty}(Y)^{N}} \varepsilon .
$$

This lemma can be proved using the same arguments as in the elliptic case (see [8] and [20]). Let us now prove the following lemma.

Lemma 7. Let $\tilde{u}_{\varepsilon}$ and $\pi_{\varepsilon, H}$ defined as in Proposition 1. There exists a constant $C>0 \varepsilon$, which does not depend on $k$ and $H$, verifying

$$
\left\|\nabla\left(\pi_{H} \tilde{u}_{\varepsilon}\left(t, \widehat{w}^{\varepsilon}(x)\right)-\pi_{\varepsilon, H} \tilde{u}_{\varepsilon}\right)\right\|_{L^{2}((0, T) \times \Omega)^{N}} \leqslant C \sqrt{\frac{\varepsilon}{H}},
$$

where $\pi_{H}$ is the interpolation operator on $V_{H}$.

Proof. The term which will be bounded is

$$
\begin{aligned}
\nabla\left(\left(\pi_{H} \tilde{u}_{\varepsilon}\right)\left(t, \widehat{w}^{\varepsilon}(x)\right)-\pi_{\varepsilon, H} \tilde{u}_{\varepsilon}\right)=\nabla( & \left(\left(\pi_{H} \tilde{u}_{\varepsilon}\right)\left(t, \widehat{w}^{\varepsilon}(x)\right)-\left(\pi_{H} \tilde{u}_{\varepsilon}\right)\left(t, \widetilde{w}^{\varepsilon, H}(x)\right)\right) \\
=\nabla \widehat{w}_{i}^{\varepsilon}(x) \partial_{x_{i}}\left(\pi_{H} \tilde{u}_{\varepsilon}\right)\left(t, \widehat{w}^{\varepsilon}(x)\right) & -\nabla \widetilde{w}_{i}^{\varepsilon, H}(x) \partial_{x_{i}}\left(\pi_{H} \tilde{u}_{\varepsilon}\right)\left(t, \widetilde{w}^{\varepsilon, H}(x)\right) .
\end{aligned}
$$

We have

$$
\begin{aligned}
& \left\|\nabla \widehat{w}_{i}^{\varepsilon} \partial_{x_{i}}\left(\pi_{H} \tilde{u}_{\varepsilon}\right) \circ \widehat{w}^{\varepsilon}-\nabla \widetilde{w}_{i}^{\varepsilon, H} \partial_{x_{i}}\left(\pi_{H} \tilde{u}_{\varepsilon}\right) \circ \widetilde{w}^{\varepsilon, H}\right\|_{L^{2}((0, T) \times \Omega)^{N}} \\
& \leqslant\left\|\left(\nabla \widehat{w}_{i}^{\varepsilon}-\nabla \widetilde{w}_{i}^{\varepsilon, H}\right) \partial_{x_{i}}\left(\pi_{H} \tilde{u}_{\varepsilon}\right) \circ \widetilde{w}^{\varepsilon, H}\right\|_{L^{2}((0, T) \times \Omega)^{N}} \\
& \quad+\left\|\nabla \widehat{w}_{i}^{\varepsilon}\left(\partial_{x_{i}}\left(\pi_{H} \tilde{u}_{\varepsilon}\right) \circ \widehat{w}^{\varepsilon}-\partial_{x_{i}}\left(\pi_{H} \tilde{u}_{\varepsilon}\right) \circ \widetilde{w}^{\varepsilon, H}\right)\right\|_{L^{2}((0, T) \times \Omega)^{N}} .
\end{aligned}
$$

Yet,

$$
\left\|\nabla\left(\widehat{w}^{\varepsilon}-\widetilde{w}^{\varepsilon, H}\right)\right\|_{L^{2}(\Omega)^{N \times N}}^{2}=\sum_{K \in \mathcal{K}_{H}}\left\|\nabla\left(\widehat{w}^{\varepsilon}-\widetilde{w}^{\varepsilon, K}\right)\right\|_{L^{2}(K)^{N \times N}}^{2} .
$$

Applying Lemma 6 in each cell $K$ gives

$$
\begin{aligned}
\left\|\nabla\left(\widehat{w}^{\varepsilon}-\widetilde{w}^{\varepsilon, H}\right)\right\|_{L^{2}(\Omega)^{N \times N}}^{2} & =\sum_{K \in \mathcal{K}_{H}}\left\|\nabla\left(\widehat{w}^{\varepsilon}-\widetilde{w}^{\varepsilon, K}\right)\right\|_{L^{2}(K)^{N \times N}}^{2} \\
& \leqslant \sum_{K \in \mathcal{K}_{H}} C \varepsilon|\partial K| \\
& \leqslant C \varepsilon H^{N-1} H^{-N},
\end{aligned}
$$

since the perimeter of a cell is on the order of $H^{N-1}$ and the number of cells to cover $\Omega$ is on the order of $H^{-N}$. Thus

$$
\left\|\nabla\left(\widehat{w}^{\varepsilon}-\widetilde{w}^{\varepsilon, H}\right)\right\|_{L^{2}(\Omega)^{N \times N}} \leqslant \sqrt{C \frac{\varepsilon}{H}} .
$$


And so

$$
\left\|\nabla\left(\widehat{w}_{i}^{\varepsilon}-\widetilde{w}_{i}^{\varepsilon, H}\right) \partial_{x_{i}}\left(\pi_{H} \tilde{u}_{\varepsilon}\right) \circ \widetilde{w}^{\varepsilon, H}\right\|_{L^{2}(\Omega)^{N}} \leqslant \sqrt{C \frac{\varepsilon}{H}}\left\|\pi_{H} u\right\|_{W^{1, \infty}(\Omega)} .
$$

Moreover, since $\left\|\pi_{H} u\right\|_{W^{1, \infty}(\Omega)}$ is bounded

$$
\left\|\nabla\left(\widehat{w}_{i}^{\varepsilon}-\widetilde{w}_{i}^{\varepsilon, H}\right) \partial_{x_{i}}\left(\pi_{H} \tilde{u}_{\varepsilon}\right) \circ \widetilde{w}^{\varepsilon, H}\right\|_{L^{2}(\Omega)^{N}} \leqslant C \sqrt{\frac{\varepsilon}{H}} .
$$

Hence

$$
\left\|\nabla\left(\widehat{w}_{i}^{\varepsilon}-\widetilde{w}_{i}^{\varepsilon, H}\right) \partial_{x_{i}}\left(\pi_{H} \tilde{u}_{\varepsilon}\right) \circ \widetilde{w}^{\varepsilon, H}\right\|_{L^{2}((0, T) \times \Omega)^{N}} \leqslant C \sqrt{\frac{\varepsilon}{H}} .
$$

To bound the second term of (4.28), a second order Taylor expansion will first be computed and the estimation (4.25) will be applied. However, this Taylor expansion is only valid if the function is $\mathcal{C}^{2}$ in the domain considered. The functions $\Phi_{l}^{H}$ and $\pi_{H} u$ are $\mathcal{C}^{\infty}$ in each cell $K$. Thus, let us define a subset of $K$ in which $\widehat{w}^{\varepsilon}$ and $\widetilde{w}^{\varepsilon, K}$ also belong to $K$. In fact, the estimation (4.26) gives

$$
\left\|\widehat{w}^{\varepsilon}-x-\left(\widetilde{w}^{\varepsilon, K}-x\right)\right\|_{L^{\infty}(K)^{N}} \leqslant\|w\|_{L^{\infty}(Y)^{N}} \varepsilon .
$$

So

$$
\left\|\widetilde{w}^{\varepsilon, K}-x\right\|_{L^{\infty}(K)^{N}} \leqslant\|w\|_{L^{\infty}(Y)^{N}} \varepsilon+\left\|\widehat{w}^{\varepsilon}-x\right\|_{L^{\infty}(K)^{N}} .
$$

Since $\widehat{w}^{\varepsilon}-x=\varepsilon w\left(\frac{x}{\varepsilon}\right)$,

$$
\left\|\widetilde{w}^{\varepsilon, K}-x\right\|_{L^{\infty}(K)^{N}} \leqslant 2\|w\|_{L^{\infty}(Y)^{N}} \varepsilon .
$$

Let us define

$$
C_{K}=\left\{x \in K \mid d(x, \partial K)>2 \varepsilon\|w\|_{L^{\infty}(Y)^{N}}\right\} .
$$

With this definition, if $x \in C_{K}$,

$$
\widehat{w}^{\varepsilon}(x) \in K
$$

and

$$
\widetilde{w}^{\varepsilon, K}(x) \in K .
$$

The second term of (4.28) will be split into two parts:

$$
\begin{aligned}
\left\|\nabla \widehat{w}_{i}^{\varepsilon}\left(\partial_{x_{i}}\left(\pi_{H} \tilde{u}_{\varepsilon}\right) \circ \widehat{w}^{\varepsilon}-\partial_{x_{i}}\left(\pi_{H} u\right) \circ \widetilde{w}^{\varepsilon, H}\right)\right\|_{L^{2}(K)^{N}}^{2} \\
=\left\|\nabla \widehat{w}_{i}^{\varepsilon}\left(\partial_{x_{i}}\left(\pi_{H} \tilde{u}_{\varepsilon}\right) \circ \widehat{w}^{\varepsilon}-\partial_{x_{i}}\left(\pi_{H} \tilde{u}_{\varepsilon}\right) \circ \widetilde{w}^{\varepsilon, H}\right)\right\|_{L^{2}\left(C_{K}\right)^{N}}^{2} \\
\quad+\left\|\nabla \widehat{w}_{i}^{\varepsilon}\left(\partial_{x_{i}}\left(\pi_{H} \tilde{u}_{\varepsilon}\right) \circ \widehat{w}^{\varepsilon}-\partial_{x_{i}}\left(\pi_{H} \tilde{u}_{\varepsilon}\right) \circ \widetilde{w}^{\varepsilon, H}\right)\right\|_{L^{2}\left(K \backslash C_{K}\right)^{N}}^{2} .
\end{aligned}
$$

In $C_{K}$, a Taylor inequality can be applied.

REMARK 4. The following inequality can be shown using interpolation results (see [13]):

$$
\begin{aligned}
\left\|\nabla^{2} \pi_{H} u\right\|_{L^{\infty}(K)^{N \times N}} & \leqslant\left\|\nabla^{2} u\right\|_{L^{\infty}(K)^{N \times N}}+\left\|\nabla^{2}\left(u-\pi_{H} u\right)\right\|_{L^{\infty}(K)^{N \times N}} \\
& \leqslant\left\|\nabla^{2} u\right\|_{L^{\infty}(K)^{N \times N}}+C H^{k-1}|u|_{W^{k+1, \infty}(K)} .
\end{aligned}
$$


Since $k \geqslant 1$,

$$
\left\|\nabla^{2} \pi_{H} u\right\|_{L^{\infty}(K)^{N \times N}}
$$

is bounded.

Then, the following inequalities are verified

$$
\begin{aligned}
&\left\|\nabla \widehat{w}_{i}^{\varepsilon}\left(\partial_{x_{i}}\left(\pi_{H} \tilde{u}_{\varepsilon}\right) \circ \widehat{w}^{\varepsilon}-\partial_{x_{i}}\left(\pi_{H} \tilde{u}_{\varepsilon}\right) \circ \widetilde{w}^{\varepsilon, H}\right)\right\|_{L^{2}\left(C_{K}\right)^{N}} \\
& \leqslant\left\|\nabla \widehat{w}^{\varepsilon}\right\|_{L^{\infty}(K)^{N \times N}}\left\|\nabla^{2}\left(\pi_{H} u\right)\right\|_{L^{\infty}(K)^{N \times N}}\left\|\widehat{w}^{\varepsilon}-\widetilde{w}^{\varepsilon, K}\right\|_{L^{2}\left(C_{K}\right)^{N}} \\
& \leqslant C\left\|\widehat{w}^{\varepsilon}-\widetilde{w}^{\varepsilon, K}\right\|_{L^{2}(K)^{N}} \\
& \leqslant C \varepsilon \sqrt{|K|} \\
& \leqslant C \varepsilon \sqrt{H^{N}}
\end{aligned}
$$

since the cell volumes are on the order of $H^{N}$. In $K \backslash C_{K}$, let us use the fact that

$$
\left|K \backslash C_{K}\right|=\left|\left\{x \in K \mid d(x, \partial K) \leqslant 2 \varepsilon\|w\|_{L^{\infty}(Y)^{N}}\right\}\right| \leqslant C|\partial K| \varepsilon .
$$

This implies that

$$
\begin{aligned}
\| \nabla \widehat{w}_{i}^{\varepsilon}\left(\partial_{x_{i}}\left(\pi_{H} \tilde{u}_{\varepsilon}\right) \circ \widehat{w}^{\varepsilon}-\partial_{x_{i}}\right. & \left.\left(\pi_{H} \tilde{u}_{\varepsilon}\right) \circ \widetilde{w}^{\varepsilon, H}\right) \|_{L^{2}\left(K \backslash C_{K}\right)^{N}} \\
& \leqslant 2\left\|\nabla \widehat{w}^{\varepsilon}\right\|_{L^{\infty}(K)^{N \times N}}\left\|\nabla\left(\pi_{H} u\right)\right\|_{L^{\infty}(K)^{N}} \sqrt{C|\partial K| \varepsilon} \\
& \leqslant C \sqrt{\varepsilon H^{N-1}} .
\end{aligned}
$$

Inserting inequalities (4.34) and (4.33) in (4.32) leads to

$$
\begin{aligned}
\left\|\nabla \widehat{w}_{i}^{\varepsilon}\left(\partial_{x_{i}}\left(\pi_{H} \tilde{u}_{\varepsilon}\right) \circ \widehat{w}^{\varepsilon}-\partial_{x_{i}}\left(\pi_{H} \tilde{u}_{\varepsilon}\right) \circ \widetilde{w}^{\varepsilon, H}\right)\right\|_{L^{2}(K)^{N}}^{2} & \leqslant C \varepsilon H^{N-1}(\varepsilon H+1) \\
& \leqslant C \varepsilon H^{N-1},
\end{aligned}
$$

because $\varepsilon$ and $H$ are bounded. Computing the $L^{2}$ norm on the whole domain gives

$$
\begin{aligned}
&\left\|\nabla \widehat{w}_{i}^{\varepsilon}\left(\partial_{x_{i}}\left(\pi_{H} \tilde{u}_{\varepsilon}\right) \circ \widehat{w}^{\varepsilon}-\partial_{x_{i}}\left(\pi_{H} \tilde{u}_{\varepsilon}\right) \circ \widetilde{w}^{\varepsilon, H}\right)\right\|_{L^{2}(\Omega)^{N}}^{2} \\
& \quad=\sum_{K \in \mathcal{K}_{H}}\left\|\nabla \widehat{w}_{i}^{\varepsilon}\left(\partial_{x_{i}}\left(\pi_{H} \tilde{u}_{\varepsilon}\right) \circ \widehat{w}^{\varepsilon}-\partial_{x_{i}}\left(\pi_{H} \tilde{u}_{\varepsilon}\right) \circ \widetilde{w}^{\varepsilon, H}\right)\right\|_{L^{2}(K)^{N}}^{2} \\
& \leqslant \sum_{K \in \mathcal{K}_{H}} C \varepsilon H^{N-1} \\
& \leqslant C \frac{\varepsilon}{H} .
\end{aligned}
$$

This implies

$$
\left\|\nabla \widehat{w}_{i}^{\varepsilon}\left(\partial_{x_{i}}\left(\pi_{H} \tilde{u}_{\varepsilon}\right) \circ \widehat{w}^{\varepsilon}-\partial_{x_{i}}\left(\pi_{H} \tilde{u}_{\varepsilon}\right) \circ \widetilde{w}^{\varepsilon, H}\right)\right\|_{L^{2}(\Omega)^{N}} \leqslant C \sqrt{\frac{\varepsilon}{H}} .
$$

Hence

$$
\left\|\nabla \widehat{w}_{i}^{\varepsilon}\left(\partial_{x_{i}}\left(\pi_{H} \tilde{u}_{\varepsilon}\right) \circ \widehat{w}^{\varepsilon}-\partial_{x_{i}}\left(\pi_{H} \tilde{u}_{\varepsilon}\right) \circ \widetilde{w}^{\varepsilon, H}\right)\right\|_{L^{2}((0, T) \times \Omega)^{N}} \leqslant C \sqrt{\frac{\varepsilon}{H}} .
$$

Thus, injecting inequalities (4.30) and (4.35) into (4.28) gives the desired result. 
4.4. The inertial term $X_{2}$.

This section provides an upper bound to the term $X_{2}$. More precisely, it aims at proving the following proposition.

Proposition 2. Let $u_{\varepsilon}$ be the solution of problem (3.1) and $\tilde{u}_{\varepsilon}$ defined by (2.5), then

$$
X_{2}=\left\|u_{\varepsilon}-\pi_{\varepsilon, H} \tilde{u}_{\varepsilon}\right\|_{L^{\infty}\left((0, T), L^{2}(\Omega)\right)} \leqslant C\left(H^{k}+\sqrt{\frac{\varepsilon}{H}}\right) .
$$

where $\pi_{\varepsilon, H}$ is the interpolation operator associated with $V_{\varepsilon, H}$ verifying (3.5).

Proof. The term $X_{2}$ can be bounded in the same way that $X_{1}$ was bounded. However, the a priori error estimate used is not (4.18), but the estimation (2.16), which leads to:

$$
\left\|u_{\varepsilon}(t, x)-u\left(t, x-\frac{b^{*} t}{\varepsilon}\right)-\varepsilon u_{1}\left(t, x-\frac{b^{*} t}{\varepsilon}, \frac{x}{\varepsilon}\right)\right\|_{L^{\infty}\left((0, T), L^{2}(\Omega)\right)} \leqslant C \varepsilon,
$$

with a constant $C>0$. The proof of Proposition 1 can then easily be adapted to prove this proposition.

4.5. The convective derivative terms $X_{3}$ and $X_{4}$.

This section is dedicated to the proof of a proposition giving an upper bounder of the terms $X_{3}$ and $X_{4}$. Let us first state this proposition.

Proposition 3. Let $u_{\varepsilon}$ be the solution of (3.1) and $\tilde{u}_{\varepsilon}$ verifying (2.5). There exists a constant $C>0$ which does not depend on $\varepsilon$ and $H$ such that

$$
\begin{aligned}
& \left\|D_{t}\left(u_{\varepsilon}-\pi_{\varepsilon, H} \tilde{u}_{\varepsilon}\right)\right\|_{L^{2}\left((0, T), \dot{H}_{\#}^{-1}(\Omega)\right)} \\
& \quad+\left\|\int_{\Omega} D_{t}\left(u_{\varepsilon}-\pi_{\varepsilon, H} \tilde{u}_{\varepsilon}\right)\right\|_{L^{2}(0, T)} \leqslant C\left(H^{k}+H^{k+1} \frac{\left|b^{*}\right|}{\varepsilon}+\frac{\left|b^{*}\right|}{\varepsilon} \varepsilon+\sqrt{\frac{\varepsilon}{H}}\right),
\end{aligned}
$$

where $\pi_{\varepsilon, H}$ is the interpolation operator on $V_{\varepsilon, H}$ defined by (3.5).

Proof. In order to prove this proposition, let us first make the following remark.

REMARK 5. For any function $f$,

$$
\|f\|_{L^{2}\left((0, T), \dot{H}_{\#}^{-1}(\Omega)\right)}
$$

and

$$
\left\|\int_{\Omega} f\right\|_{L^{2}((0, T))}
$$

can be bounded using similar arguments. Indeed, by definition of this norm

$$
\|f\|_{\dot{H}_{\#}^{-1}(\Omega)}=\max _{\varphi \in \dot{H}_{\#}^{1}(\Omega) \backslash\{0\}} \frac{\int_{\Omega} f \varphi}{\|\nabla \varphi\|_{L^{2}(\Omega)^{N}}} .
$$

Using Poincaré inequality the semi-norm $|\varphi|_{H^{1}(\Omega)}$ is equivalent to the norm $\|\varphi\|_{H^{1}(\Omega)}$. And, it can be noted that

$$
\frac{\left|\int_{\Omega} f \varphi\right|}{\|\varphi\|_{H^{1}(\Omega)}}=\frac{1}{|\Omega|^{\frac{1}{2}}}\left|\int_{\Omega} f\right| \quad \text { if } \varphi=1 .
$$

Therefore, in the following, the term $\frac{\left|\int_{\Omega} f \varphi\right|}{\|\varphi\|_{H^{1}(\Omega)}}$ will be bounded. In this section, only the case $\varphi \in \dot{H}_{\#}^{1}(\Omega)$ will be considered. However, the properties are still valid in the case $\varphi=1$. 
Moreover, using once more Poincaré and Cauchy-Schwarz inequalities, any function $\varphi \in \dot{H}_{\#}^{1}(\Omega)$ verifies

$$
\frac{\left|\int_{\Omega} f \varphi\right|}{\|\nabla \varphi\|_{L^{2}(\Omega)^{N}}} \leqslant C_{\Omega} \frac{\left|\int_{\Omega} f \varphi\right|}{\|\varphi\|_{L^{2}(\Omega)}} \leqslant C_{\Omega}\|f\|_{L^{2}(\Omega)},
$$

where $C_{\Omega}$ is the constant associated to the Poincaré inequality applied in $\Omega$. Hence

$$
\|u\|_{\dot{H}^{-1}(\Omega)} \leqslant C_{\Omega}\|u\|_{L^{2}(\Omega)} .
$$

Using this remark we will only prove here the inequality

$$
\left\|D_{t}\left(u_{\varepsilon}-\pi_{\varepsilon, H} \tilde{u}_{\varepsilon}\right)\right\|_{L^{2}\left((0, T), \dot{H}^{-1}(\Omega)\right)} \leqslant C\left(H^{k}+H^{k+1} \frac{\left|b^{*}\right|}{\varepsilon}+\frac{\left|b^{*}\right|}{\varepsilon} \varepsilon+\sqrt{\frac{\varepsilon}{H}}\right),
$$

As in Section 4.3, the term to be bounded is split into three:

$$
\left\|D_{t}\left(u_{\varepsilon}-\pi_{\varepsilon, H} \tilde{u}_{\varepsilon}\right)\right\|_{L^{2}\left((0, T), \dot{H}^{-1}(\Omega)_{\#}\right)} \leqslant D_{1}+D_{2}+D_{3},
$$

where

$$
\begin{aligned}
D_{1} & =\left\|D_{t} u_{\varepsilon}-D_{t}\left(\tilde{u}_{\varepsilon}(t, \cdot) \circ \widehat{w}^{\varepsilon}\right)\right\|_{L^{2}\left((0, T), \dot{H}_{\#}^{-1}(\Omega)\right)}, \\
D_{2} & =\left\|D_{t}\left(\left(\tilde{u}_{\varepsilon}-\pi_{H} \tilde{u}_{\varepsilon}\right)(t, \cdot) \circ \widehat{w}^{\varepsilon}\right)\right\|_{L^{2}\left((0, T), \dot{H}_{\#}^{-1}(\Omega)\right)}, \\
\text { and } \quad D_{3} & =\left\|D_{t}\left(\pi_{H} \tilde{u}_{\varepsilon}(t, \cdot) \circ \widehat{w}^{\varepsilon}-\pi_{\varepsilon, H} \tilde{u}_{\varepsilon}\right)\right\|_{L^{2}\left((0, T), \dot{H}_{\#}^{-1}(\Omega)\right)} .
\end{aligned}
$$

$D_{1}$ is a global homogenization term bounded in Section 4.5.1. $D_{2}$ is an interpolation term on the coarse mesh to which an upper bound is given inside Section 4.5.2. Section 4.5.3 is dedicated to bounding $D_{3}$ applying homogenization results in each coarse cell.

Injecting the results of Lemmata 8, 10 and 12, or more precisely, the inequalities (4.41), (4.49) and (4.57) into inequality (4.40), we get:

$$
\left\|D_{t}\left(u_{\varepsilon}-\pi_{\varepsilon, H} \tilde{u}_{\varepsilon}\right)\right\|_{L^{2}\left((0, T), \dot{H}^{-1}(\Omega)\right)} \leqslant C\left(\varepsilon+H^{k}+H^{k+1} \frac{\left|b^{*}\right|}{\varepsilon}+\frac{\left|b^{*}\right|}{\varepsilon} \varepsilon+\sqrt{\frac{\varepsilon}{H}}\right) .
$$

Providing that $\varepsilon \leqslant C \sqrt{\frac{\varepsilon}{H}}$, the proposition is then proved.

\subsubsection{The global homogenization term $D_{1}$.}

Let us prove the following lemma

Lemma 8. Let $u_{\varepsilon}$ be the solution of problem (3.1) and $\tilde{u}_{\varepsilon}$ verifying (2.5). There exists a constant $C>0$ which does not depend on $\varepsilon$ and $H$ such that

$$
\left\|D_{t} u_{\varepsilon}-D_{t}\left(\tilde{u}_{\varepsilon}(t, \cdot) \circ \widehat{w}^{\varepsilon}\right)\right\|_{L^{2}\left((0, T), \dot{H}_{\#}^{-1}(\Omega)\right)} \leqslant C \varepsilon .
$$

This proof requires the following classical lemma which will not be proved here.

Lemma 9. Let $g \in L_{\#}^{2}(Y)$ such that $\int_{Y} g(y) d y=0$. There exists a function $\zeta \in H_{\#}^{1}(Y)^{N}$ such that

$$
\left\{\begin{array}{ccc}
-\operatorname{div}_{y} \zeta & = & g(y), \\
\int_{Y} \zeta(y) d y & = & 0, \\
y \mapsto \zeta(y) \text { is } Y \text {-periodic. } &
\end{array}\right.
$$



function

Proof of Lemma 8 In order to bound $\left\|D_{t} u_{\varepsilon}-D_{t}\left(\tilde{u}_{\varepsilon}(t, \cdot) \circ \widehat{w}^{\varepsilon}\right)\right\|_{L^{2}\left((0, T), \dot{H}_{\#}^{-1}(\Omega)\right)}$, let us consider a test

$$
\varphi \in L^{2}\left((0, T), \dot{H}_{\#}^{1}(\Omega)\right)
$$

The aim is now to bound the integral

$$
\int_{\Omega} D_{t}\left(u_{\varepsilon}-\tilde{u}_{\varepsilon}(t, \cdot) \circ \widehat{w}^{\varepsilon}\right) \varphi d x
$$

Recalling that $D_{t}=\rho^{\varepsilon} \partial_{t}+\frac{1}{\varepsilon} b^{\varepsilon} \cdot \nabla$,

$$
D_{t} u_{\varepsilon}=\operatorname{div}\left(A^{\varepsilon} \nabla u_{\varepsilon}\right)
$$

First, we have

$$
\begin{aligned}
\rho^{\varepsilon}(x) \partial_{t}\left(\tilde{u}_{\varepsilon}(t, \cdot) \circ \widehat{w}^{\varepsilon}\right)(x)=\rho^{\varepsilon}(x) \partial_{t}(u & \left.\left(t, x+\varepsilon w\left(\frac{x}{\varepsilon}\right)-\frac{b^{*} t}{\varepsilon}\right)\right) \\
=\rho^{\varepsilon}(x)\left(\partial_{t} u\right) & \left(t, x+\varepsilon w\left(\frac{x}{\varepsilon}\right)-\frac{b^{*} t}{\varepsilon}\right) \\
& -\frac{1}{\varepsilon} \rho^{\varepsilon}(x) b^{*} \cdot(\nabla u)\left(t, x+\varepsilon w\left(\frac{x}{\varepsilon}\right)-\frac{b^{*} t}{\varepsilon}\right) .
\end{aligned}
$$

Moreover

$$
\begin{aligned}
\nabla\left(\tilde{u}_{\varepsilon}(t, \cdot) \circ \widehat{w}^{\varepsilon}\right)(x) & =\nabla\left(u\left(t, x+\varepsilon w\left(\frac{x}{\varepsilon}\right)-\frac{b^{*} t}{\varepsilon}\right)\right) \\
& =\left(e_{i}+\nabla_{y} w_{i}\left(\frac{x}{\varepsilon}\right)\right) \partial_{x_{i}} u\left(t, x+\varepsilon w\left(\frac{x}{\varepsilon}\right)-\frac{b^{*} t}{\varepsilon}\right) .
\end{aligned}
$$

Thus

$$
\begin{aligned}
D_{t}\left(\tilde{u}_{\varepsilon}(t, \cdot) \circ \widehat{w}^{\varepsilon}\right)= & \rho^{\varepsilon}(x)\left(\partial_{t} u\right)\left(t, x+\varepsilon w\left(\frac{x}{\varepsilon}\right)-\frac{b^{*} t}{\varepsilon}\right) \\
& -\frac{1}{\varepsilon} \rho^{\varepsilon}(x) b^{*} \cdot(\nabla u)\left(t, x+\varepsilon w\left(\frac{x}{\varepsilon}\right)-\frac{b^{*} t}{\varepsilon}\right) \\
& +\frac{1}{\varepsilon} b^{\varepsilon} \cdot\left(e_{i}+\nabla_{y} w_{i}\left(\frac{x}{\varepsilon}\right)\right) \partial_{x_{i}} u\left(t, x+\varepsilon w\left(\frac{x}{\varepsilon}\right)-\frac{b^{*} t}{\varepsilon}\right) .
\end{aligned}
$$

Let us define

$$
\begin{aligned}
& I_{1}=-\frac{1}{\varepsilon} \rho^{\varepsilon}(x) b^{*} \cdot(\nabla u)\left(t, x+\varepsilon w\left(\frac{x}{\varepsilon}\right)-\frac{b^{*} t}{\varepsilon}\right) \\
& I_{2}=\frac{1}{\varepsilon} b^{\varepsilon} \cdot\left(I d+\nabla_{y} w_{i}\left(\frac{x}{\varepsilon}\right)\right) \partial_{x_{i}} u\left(t, x+\varepsilon w\left(\frac{x}{\varepsilon}\right)-\frac{b^{*} t}{\varepsilon}\right) .
\end{aligned}
$$


Using the fact that $u$ verifies (2.2), we have

$$
\begin{aligned}
\rho^{\varepsilon}(x)\left(\partial_{t} u\right)\left(t, x+\varepsilon w\left(\frac{x}{\varepsilon}\right)-\frac{b^{*} t}{\varepsilon}\right)= & \rho^{*}\left(\partial_{t} u\right)\left(t, x+\varepsilon w\left(\frac{x}{\varepsilon}\right)-\frac{b^{*} t}{\varepsilon}\right) \\
& +\left(\rho^{\varepsilon}(x)-\rho^{*}\right)\left(\partial_{t} u\right)\left(t, x+\varepsilon w\left(\frac{x}{\varepsilon}\right)-\frac{b^{*} t}{\varepsilon}\right) \\
= & \operatorname{div}\left(A^{*}(\nabla u)\right)\left(t, x+\varepsilon w\left(\frac{x}{\varepsilon}\right)-\frac{b^{*} t}{\varepsilon}\right) \\
& +\left(\rho^{\varepsilon}(x)-\rho^{*}\right)\left(\partial_{t} u\right)\left(t, x+\varepsilon w\left(\frac{x}{\varepsilon}\right)-\frac{b^{*} t}{\varepsilon}\right) .
\end{aligned}
$$

Introducing

$$
\begin{aligned}
I_{3} & =\operatorname{div}\left(A^{*}(\nabla u)\right)\left(t, x+\varepsilon w\left(\frac{x}{\varepsilon}\right)-\frac{b^{*} t}{\varepsilon}\right), \\
\text { and } \quad I_{4} & =\left(\rho^{\varepsilon}(x)-\rho^{*}\right)\left(\partial_{t} u\right)\left(t, x+\varepsilon w\left(\frac{x}{\varepsilon}\right)-\frac{b^{*} t}{\varepsilon}\right),
\end{aligned}
$$

the convective derivative can be rewritten in

$$
D_{t}\left(\tilde{u}_{\varepsilon}(t, \cdot) \circ \widehat{w}^{\varepsilon}\right)=I_{1}+I_{2}+I_{3}+I_{4} .
$$

Applying Lemma 9, the term $I_{4}$ can be bounded. Indeed, since $\int_{Y}\left(\rho(y)-\rho^{*}\right) d y=0$, there exists a function $\zeta \in H_{\#}^{1}(Y)^{N}$ such that $-\operatorname{div}_{y} \zeta(y)=\rho(y)-\rho^{*}$. Then, we have

$$
\begin{aligned}
\int_{\Omega}\left(\rho^{\varepsilon}(x)-\rho^{*}\right) & \left(\partial_{t} u\right)\left(t, x+\varepsilon w\left(\frac{x}{\varepsilon}\right)-\frac{b^{*} t}{\varepsilon}\right) \varphi(x) d x \\
& =-\int_{\Omega} \operatorname{div}_{y} \zeta\left(\frac{x}{\varepsilon}\right)\left(\partial_{t} u\right)\left(t, x+\varepsilon w\left(\frac{x}{\varepsilon}\right)-\frac{b^{*} t}{\varepsilon}\right) \varphi(x) d x \\
& =-\int_{\Omega} \varepsilon \operatorname{div}\left(\zeta\left(\frac{x}{\varepsilon}\right)\right)\left(\partial_{t} u\right)\left(t, x+\varepsilon w\left(\frac{x}{\varepsilon}\right)-\frac{b^{*} t}{\varepsilon}\right) \varphi(x) d x \\
& =\int_{\Omega} \varepsilon \zeta\left(\frac{x}{\varepsilon}\right) \cdot \nabla\left(\left(\partial_{t} u\right)\left(t, x+\varepsilon w\left(\frac{x}{\varepsilon}\right)-\frac{b^{*} t}{\varepsilon}\right) \varphi(x)\right) d x,
\end{aligned}
$$

using integrations by parts and the periodicity of the different functions (see Remark 2). Since $\partial_{t} u=$ $\frac{1}{\rho^{*}} \operatorname{div}\left(A^{*} \nabla u\right)$ and $u \in L^{\infty}\left((0, T), W^{k+3, \infty}(\Omega)\right)$,

$$
\partial_{t} u \in L^{\infty}\left((0, T), W^{k+1, \infty}(\Omega)\right) \subset L^{\infty}\left((0, T), W^{1, \infty}(\Omega)\right) .
$$

Moreover, $\zeta \in L_{\#}^{2}(Y)^{N}$ so $\zeta(\dot{\bar{\varepsilon}}) \in L^{2}(\Omega)^{N}$ and applying Cauchy-Schwarz inequality

$$
\begin{aligned}
& \left|\int_{\Omega} \varepsilon \zeta\left(\frac{x}{\varepsilon}\right) \cdot \nabla\left(\left(\partial_{t} u\right)\left(t, x+\varepsilon w\left(\frac{x}{\varepsilon}\right)-\frac{b^{*} t}{\varepsilon}\right) \varphi(x)\right) d x\right| \\
& \quad \leqslant \varepsilon\left\|\zeta\left(\frac{\cdot}{\varepsilon}\right)\right\|_{L^{2}(\Omega)^{N}}\left\|\nabla\left(\left(\partial_{t} u\right)\left(t, x+\varepsilon w\left(\frac{x}{\varepsilon}\right)-\frac{b^{*} t}{\varepsilon}\right) \varphi(x)\right)\right\|_{L^{\infty}\left((0, T), L^{2}(\Omega)\right)^{N}} \\
& \quad \leqslant \varepsilon\left\|\zeta\left(\frac{\dot{\varepsilon}}{\varepsilon}\right)\right\|_{L^{2}(\Omega)^{N}}\left\|\partial_{t} u\right\|_{L^{\infty}\left((0, T), W^{1, \infty}(\Omega)\right)}\|\varphi\|_{H^{1}(\Omega)} .
\end{aligned}
$$

This proves that

$$
\left\|I_{4}\right\|_{L^{\infty}\left((0, T), \dot{H}_{\#}^{-1}(\Omega)\right)} \leqslant C \varepsilon .
$$


Applying Taylor expansions to the terms $I_{1}, I_{2}$ and $I_{3}$ and using the fact that $u \in L^{\infty}\left((0, T), W^{3, \infty}(\Omega)\right)$, we have:

$$
\begin{aligned}
& I_{1}=-\frac{1}{\varepsilon} \rho^{\varepsilon}(x) b^{*} \cdot(\nabla u)(t,\left.-\frac{b^{*} t}{\varepsilon}\right)-\rho^{\varepsilon}(x) b^{*} \cdot \nabla \partial_{x_{i}} u\left(t, x-\frac{b^{*} t}{\varepsilon}\right) w_{i}\left(\frac{x}{\varepsilon}\right) \\
&+\varepsilon \int_{0}^{1} w_{i}\left(\frac{x}{\varepsilon}\right) w_{j}\left(\frac{x}{\varepsilon}\right) \rho^{\varepsilon}(x) b^{*} \cdot \nabla \partial_{x_{i}, x_{j}}^{2} u\left(t, x-\frac{b^{*} t}{\varepsilon}+\varepsilon s w\left(\frac{x}{\varepsilon}\right)\right)(1-s) d s, \\
& I_{2}=\frac{1}{\varepsilon} b^{\varepsilon} \cdot\left(I d+\nabla_{y} w_{i}\left(\frac{x}{\varepsilon}\right)\right) \partial_{x_{i}} u\left(t, x-\frac{b^{*} t}{\varepsilon}\right) \\
&+b^{\varepsilon} \cdot\left(I d+\nabla_{y} w_{i}\left(\frac{x}{\varepsilon}\right)\right) w_{j}\left(\frac{x}{\varepsilon}\right) \partial_{x_{i}, x_{j}}^{2} u\left(t, x-\frac{b^{*} t}{\varepsilon}\right) \\
&+\varepsilon \int_{0}^{1} b^{\varepsilon} \cdot\left(I d+\nabla_{y} w_{i}\left(\frac{x}{\varepsilon}\right)\right) w_{j}\left(\frac{x}{\varepsilon}\right) w_{k}\left(\frac{x}{\varepsilon}\right) \\
& \partial_{x_{i}, x_{j}, x_{k}}^{3} u\left(t, x-\frac{b^{*} t}{\varepsilon}+\varepsilon s w\left(\frac{x}{\varepsilon}\right)\right)(1-s) d s,
\end{aligned}
$$

and

$$
\begin{aligned}
I_{3}=\operatorname{div}\left(A^{*}(\nabla u)\right)\left(t, x-\frac{b^{*} t}{\varepsilon}\right) & \\
& +\varepsilon \int_{0}^{1} w_{i}\left(\frac{x}{\varepsilon}\right) \operatorname{div}\left(A^{*}\left(\nabla \partial_{x_{i}} u\right)\right)\left(t, x-\frac{b^{*} t}{\varepsilon}+\varepsilon s w\left(\frac{x}{\varepsilon}\right)\right) d s .
\end{aligned}
$$

This implies that

$$
\begin{aligned}
D_{t}\left(u_{\varepsilon}-\tilde{u}_{\varepsilon}(t, \cdot) \circ \widehat{w}^{\varepsilon}\right)=\operatorname{div} & \left(A^{\varepsilon} \nabla u_{\varepsilon}-A^{*} \nabla \tilde{u}_{\varepsilon}\right) \\
& +\frac{1}{\varepsilon} \rho^{\varepsilon}(x) b^{*} \cdot \nabla \tilde{u}_{\varepsilon}+\rho^{\varepsilon}(x) b^{*} \cdot \nabla \partial_{x_{i}} \tilde{u}_{\varepsilon} w_{i}\left(\frac{x}{\varepsilon}\right) \\
-\frac{1}{\varepsilon} b^{\varepsilon} \cdot\left(e_{i}+\nabla_{y} w_{i}\left(\frac{x}{\varepsilon}\right)\right) \partial_{x_{i}} \tilde{u}_{\varepsilon} & -b^{\varepsilon} \cdot\left(I d+\nabla_{y} w_{i}\left(\frac{x}{\varepsilon}\right)\right) w_{j}\left(\frac{x}{\varepsilon}\right) \partial_{x_{i}, x_{j}}^{2} \tilde{u}_{\varepsilon} \\
& +\varepsilon C(t, x)-I_{4} \\
=\operatorname{div}( & \left.\left(\left(A\left(I d+\nabla_{y} w\right)\right)\left(\frac{x}{\varepsilon}\right)-A^{*}\right) \nabla \tilde{u}_{\varepsilon}\right) \\
& \quad+\frac{1}{\varepsilon} \rho^{\varepsilon}(x) b^{*} \cdot \nabla \tilde{u}_{\varepsilon}+\rho^{\varepsilon}(x) b^{*} \cdot \nabla \partial_{x_{i}} \tilde{u}_{\varepsilon} w_{i}\left(\frac{x}{\varepsilon}\right) \\
& -\frac{1}{\varepsilon} b^{\varepsilon} \cdot\left(e_{i}+\nabla_{y} w_{i}\left(\frac{x}{\varepsilon}\right)\right) \partial_{x_{i}} \tilde{u}_{\varepsilon} \\
& \quad-b^{\varepsilon} \cdot\left(e_{i}+\nabla_{y} w_{i}\left(\frac{x}{\varepsilon}\right)\right) w_{j}\left(\frac{x}{\varepsilon}\right) \partial_{x_{i}, x_{j}}^{2} \tilde{u}_{\varepsilon} \\
+ & \operatorname{div}\left(A^{\varepsilon} \nabla u_{\varepsilon}-\left(A\left(I d+\nabla_{y} w\right)\right)\left(\frac{x}{\varepsilon}\right) \nabla \tilde{u}_{\varepsilon}\right) \\
+ & \varepsilon C(t, x)-I_{4},
\end{aligned}
$$

where $\|C\|_{L^{\infty}((0, T) \times \Omega)}$ does not depend on $\varepsilon$ because $u \in W^{3, \infty}(\Omega), w_{i} \in W^{1, \infty}(\Omega), \rho^{\varepsilon} \in L^{\infty}(\Omega)$ and 
$b^{\varepsilon} \in L^{\infty}(\Omega)^{N}$. Let us define

$$
\begin{aligned}
I_{\varepsilon}(t, x)=\operatorname{div}\left(\left(\left(A\left(I d+\nabla_{y} w\right)\right)\right.\right. & \left.\left.\left(\frac{x}{\varepsilon}\right)-A^{*}\right) \nabla \tilde{u}_{\varepsilon}\right) \\
+ & \frac{1}{\varepsilon} \rho^{\varepsilon}(x) b^{*} \cdot \nabla \tilde{u}_{\varepsilon}+\rho^{\varepsilon}(x) b^{*} \cdot \nabla \partial_{x_{i}} \tilde{u}_{\varepsilon} w_{i}\left(\frac{x}{\varepsilon}\right) \\
& -\frac{1}{\varepsilon} b^{\varepsilon} \cdot\left(e_{i}+\nabla_{y} w_{i}\left(\frac{x}{\varepsilon}\right)\right) \partial_{x_{i}} \tilde{u}_{\varepsilon}-b^{\varepsilon} \cdot\left(e_{i}+\nabla_{y} w_{i}\left(\frac{x}{\varepsilon}\right)\right) w_{j}\left(\frac{x}{\varepsilon}\right) \partial_{x_{i}, x_{j}}^{2} \tilde{u}_{\varepsilon}
\end{aligned}
$$

and

$$
J_{\varepsilon, \varphi}=\int_{\Omega} \operatorname{div}\left(A^{\varepsilon} \nabla u_{\varepsilon}-A\left(I d+\nabla_{y} w\right)\left(\frac{x}{\varepsilon}\right) \nabla \tilde{u}_{\varepsilon}\right) \varphi .
$$

We obtain

$$
\int_{\Omega} D_{t}\left(u_{\varepsilon}-\tilde{u}_{\varepsilon}(t, \cdot) \circ \widehat{w}^{\varepsilon}\right) \varphi=\int_{\Omega} I_{\varepsilon} \varphi+J_{\varepsilon, \varphi}+\varepsilon \int_{\Omega} C(t, x) \varphi-\int_{\Omega} I_{4} \varphi .
$$

We have

$$
\left|\int_{\Omega} C(t, x) \varphi\right| \leqslant \sqrt{|\Omega|}\|C\|_{L^{\infty}((0, T) \times \Omega)}\|\varphi\|_{L^{2}(\Omega)} \leqslant C_{\Omega}\|C\|_{L^{\infty}((0, T) \times \Omega)}|\varphi|_{H^{1}(\Omega)} .
$$

Now, let us focus on the term $\int_{\Omega} I_{\varepsilon} \varphi$. Let us first notice that

$$
\begin{aligned}
\operatorname{div}\left(\left(A\left(I d+\nabla_{y} w\right)\right)\left(\frac{x}{\varepsilon}\right) \nabla \tilde{u}_{\varepsilon}\right) & \\
& =\left(A\left(I d+\nabla_{y} w\right)\right)\left(\frac{x}{\varepsilon}\right): \nabla^{2} \tilde{u}_{\varepsilon}+\frac{1}{\varepsilon} \operatorname{div}_{y}\left(\left(A\left(I d+\nabla_{y} w\right)\right)\left(\frac{x}{\varepsilon}\right) \nabla \tilde{u}_{\varepsilon}\right),
\end{aligned}
$$

where : is a tensor contraction between two matrices. Moreover, in (4.45), $\tilde{u}_{\varepsilon}$ does not depend on $y$. Thus, the term multiplied by $\frac{1}{\varepsilon}$ in $I_{\varepsilon}$ is

$$
\begin{aligned}
\frac{\partial u}{\partial x_{i}}\left(t, x-\frac{b^{*} t}{\varepsilon}\right)\left(\operatorname{div}_{y}\left(\left(A\left(I d+\nabla_{y} w\right)\right)\left(\frac{x}{\varepsilon}\right) e_{i}\right)\right. & \\
& \left.+\rho\left(\frac{x}{\varepsilon}\right) b^{*} \cdot e_{i}-b^{\varepsilon} \cdot\left(e_{i}+\nabla_{y} w_{i}\left(\frac{x}{\varepsilon}\right)\right)\right)=0
\end{aligned}
$$

using the cell problem (2.12). $I_{\varepsilon}$ can therefore be rewritten in

$$
\begin{aligned}
& I_{\varepsilon}=\left(A\left(I d+\nabla_{y} w\right)\right)\left(\frac{x}{\varepsilon}\right): \nabla^{2} \tilde{u}_{\varepsilon}- A^{*}: \nabla^{2} \tilde{u}_{\varepsilon}+\rho\left(\frac{x}{\varepsilon}\right) b^{*} \cdot \nabla \partial_{x_{i}} \tilde{u}_{\varepsilon} w_{i}\left(\frac{x}{\varepsilon}\right) \\
&-b^{\varepsilon} \cdot\left(e_{i}+\nabla_{y} w_{i}\left(\frac{x}{\varepsilon}\right)\right) w_{j}\left(\frac{x}{\varepsilon}\right) \partial_{x_{i}, x_{j}}^{2} \tilde{u}_{\varepsilon} \\
&=\left(A_{i, j}+A_{i, k} \partial_{y_{k}} w_{j}\left(\frac{x}{\varepsilon}\right)+\left(\rho\left(\frac{x}{\varepsilon}\right) b_{j}^{*}-b_{j}\left(\frac{x}{\varepsilon}\right)\right) w_{i}\left(\frac{x}{\varepsilon}\right)\right. \\
&\left.-b_{k}\left(\frac{x}{\varepsilon}\right) \partial_{y_{k}} w_{i}\left(\frac{x}{\varepsilon}\right) w_{j}\left(\frac{x}{\varepsilon}\right)-A_{i, j}^{*}\right): \partial_{x_{i}, x_{j}}^{2} \tilde{u}_{\varepsilon} .
\end{aligned}
$$

Applying the definition of $A^{*}$ (see (2.3)), we have

$$
\int_{Y}\left(A_{i, j}+A_{i, k} \partial_{y_{k}} w_{j}\left(\frac{x}{\varepsilon}\right)+\left(\rho\left(\frac{x}{\varepsilon}\right) b_{j}^{*}-b_{j}\left(\frac{x}{\varepsilon}\right)\right) w_{i}\left(\frac{x}{\varepsilon}\right)\right) d y=A_{i, j}^{*} .
$$


The following term is still to be studied:

$$
b_{k}\left(\frac{x}{\varepsilon}\right) \partial_{y_{k}} w_{i}\left(\frac{x}{\varepsilon}\right) w_{j}\left(\frac{x}{\varepsilon}\right) \partial_{x_{i}, x_{j}}^{2} \tilde{u}_{\varepsilon} .
$$

Since $\frac{\partial^{2} u}{\partial x_{j} \partial x_{l}}=\frac{\partial^{2} u}{\partial x_{l} \partial x_{j}}$, we have

$$
\begin{aligned}
b_{k}\left(\frac{x}{\varepsilon}\right) \partial_{y_{k}} w_{i}\left(\frac{x}{\varepsilon}\right) & w_{j}\left(\frac{x}{\varepsilon}\right) \partial_{x_{i}, x_{j}}^{2} \tilde{u}_{\varepsilon} \\
& =\frac{1}{2} b_{k}\left(\partial_{y_{k}} w_{i}\left(\frac{x}{\varepsilon}\right) w_{j}\left(\frac{x}{\varepsilon}\right)+\partial_{y_{k}} w_{j}\left(\frac{x}{\varepsilon}\right) w_{j}\left(\frac{x}{\varepsilon}\right)\right) \partial_{x_{i}, x_{j}}^{2} \tilde{u}_{\varepsilon} \\
& =\frac{1}{2} b_{k} \partial_{y_{k}}\left(w_{i} w_{j}\right) \partial_{x_{i}, x_{j}}^{2} \tilde{u}_{\varepsilon} .
\end{aligned}
$$

The functions $w$ and $b$ being $Y$-periodic,

$$
\frac{1}{2} \int_{Y} b_{k}(y) \frac{\partial}{\partial y_{k}}\left(w_{i} w_{j}\right)(y)=-\frac{1}{2} \int_{Y} \operatorname{div}(b) w_{i} w_{j}=0,
$$

because $\operatorname{div}(b)=0 . I_{\varepsilon}$ can then be rewritten in

$$
I_{\varepsilon}=P_{i, j}^{\varepsilon}\left(\frac{x}{\varepsilon}\right) \partial_{x_{i}, x_{j}}^{2} u\left(t, x-\frac{b^{*} t}{\varepsilon}\right),
$$

with

$$
\int_{Y} P_{i, j}^{\varepsilon}(y) d y=0 .
$$

Applying Lemma 9 for each $i, j$. Therefore, the functions $P_{i, j}^{\varepsilon}$ can be rewritten in

$$
P_{i, j}^{\varepsilon}\left(\frac{x}{\varepsilon}\right)=\operatorname{div}_{x}\left(\varepsilon Z_{i, j}\left(\frac{x}{\varepsilon}\right)\right)
$$

with $Z_{i, j} \in L^{2}(Y)^{N}$. So, for $\varphi \in \dot{H}_{\#}^{1}(\Omega)$,

$$
\begin{aligned}
\int_{\Omega} I_{\varepsilon} \varphi & =\int_{\Omega} \operatorname{div}_{x}\left(\varepsilon Z_{i, j}\left(\frac{x}{\varepsilon}\right)\right) \partial_{x_{i}, x_{j}}^{2} u\left(t, x-\frac{b^{*} t}{\varepsilon}\right) \varphi(x) d x \\
& =-\int_{\Omega} \varepsilon Z_{i, j}\left(\frac{x}{\varepsilon}\right) \cdot \nabla\left(\varphi(x) \partial_{x_{i}, x_{j}}^{2} u\left(t, x-\frac{b^{*} t}{\varepsilon}\right)\right) d x .
\end{aligned}
$$

Then, since the functions $Z_{i, j}$ are bounded, we have

$$
\left|\int_{\Omega} I_{\varepsilon} \varphi\right| \leqslant C \varepsilon\|\varphi\|_{H^{1}(\Omega)}\|u\|_{L^{\infty}\left((0, T), W^{3, \infty}(\Omega)\right)} .
$$

The term $\left|J_{\varepsilon, \varphi}\right|$ has to be bounded. Let us recall that

$$
J_{\varepsilon, \varphi}=\int_{\Omega} \operatorname{div}\left(A^{\varepsilon} \nabla u_{\varepsilon}-A\left(I d+\nabla_{y} w\right)\left(\frac{x}{\varepsilon}\right) \nabla \tilde{u}_{\varepsilon}\right) \varphi .
$$

So, integrating by parts,

$$
J_{\varepsilon, \varphi}=-\int_{\Omega}\left(A^{\varepsilon} \nabla u_{\varepsilon}-A\left(I d+\nabla_{y} w\right)\left(\frac{x}{\varepsilon}\right) \nabla \tilde{u}_{\varepsilon}\right) \cdot \nabla \varphi .
$$


Let us first prove that:

$$
\left\|A^{\varepsilon} \nabla u_{\varepsilon}-A\left(I d+\nabla_{y} w\right)\left(\frac{x}{\varepsilon}\right) \nabla \tilde{u}_{\varepsilon}\right\|_{L^{2}(\Omega)^{N}} \leqslant C \varepsilon .
$$

In order to obtain this inequality, we use (4.18). This leads to

$$
\left\|\nabla u_{\varepsilon}-\left(\nabla \tilde{u}_{\varepsilon}+\varepsilon \nabla\left(u_{1}\left(t, x-\frac{b^{*} t}{\varepsilon}, \frac{x}{\varepsilon}\right)\right)\right)\right\|_{L^{2}(\Omega)^{N}} \leqslant C \varepsilon
$$

This can rewritten in

$$
\nabla u_{\varepsilon}=\nabla \tilde{u}_{\varepsilon}+\nabla_{y} u_{1}\left(t, x-\frac{b^{*} t}{\varepsilon}, \frac{x}{\varepsilon}\right)+\varepsilon \nabla_{x} u_{1}\left(t, x-\frac{b^{*} t}{\varepsilon}, \frac{x}{\varepsilon}\right)+g_{\varepsilon},
$$

with

$$
\left\|g_{\varepsilon}\right\|_{L^{2}(\Omega)^{N}} \leqslant C \varepsilon .
$$

Multiplying inequality (4.47) by $A^{\varepsilon}$, and using the definition of $u_{1}$ in (2.13), we get

$$
A^{\varepsilon} \nabla u_{\varepsilon}-A\left(I d+\nabla_{y} w\right)\left(\frac{x}{\varepsilon}\right) \nabla \tilde{u}_{\varepsilon}=\varepsilon A^{\varepsilon} w_{i}\left(\frac{x}{\varepsilon}\right) \nabla \partial_{x_{i}} u\left(t, x-\frac{b^{*} t}{\varepsilon}\right)+A^{\varepsilon} g_{\varepsilon} .
$$

The functions $w$ and the matrix $A$ being bounded, we have

$$
\left\|A^{\varepsilon} w\left(\frac{x}{\varepsilon}\right) \cdot \nabla^{2} u\left(t, x-\frac{b^{*} t}{\varepsilon}\right)\right\|_{L^{2}(\Omega)^{N}} \leqslant\|w\|_{L^{\infty}(Y)^{N}} C_{b n d}\left\|\nabla^{2} u\right\|_{L^{2}(\Omega)^{N \times N}} \leqslant C
$$

because $u \in L^{\infty}\left((0, T), W^{2, \infty}(\Omega)\right)$. Moreover, using the properties of $g_{\varepsilon}$ and the boundedness of $A^{\varepsilon}$

$$
\left\|A^{\varepsilon} g_{\varepsilon}\right\|_{L^{2}(\Omega)^{N}} \leqslant C_{b n d} C \varepsilon .
$$

This proves that

$$
\left\|A^{\varepsilon} \nabla u_{\varepsilon}-A\left(I d+\nabla_{y} w\right)\left(\frac{x}{\varepsilon}\right) \nabla \tilde{u}_{\varepsilon}\right\|_{L^{2}(\Omega)^{N}} \leqslant C \varepsilon .
$$

Applying Cauchy-Schwarz inequality, this leads to

$$
\left|J_{\varepsilon, \varphi}\right|=\left|\int_{\Omega}\left(A^{\varepsilon}-\left(A\left(I d+\nabla_{y} w\right)\right)\left(\frac{x}{\varepsilon}\right)\right) \nabla \tilde{u}_{\varepsilon} \cdot \nabla \varphi\right| \leqslant C \varepsilon|\varphi|_{H^{1}(\Omega)} .
$$

Then, injecting inequalities (4.42), (4.44), (4.46) and (4.48) into (4.43) gives

$$
\left|\int_{\Omega} D_{t}\left(u_{\varepsilon}-\tilde{u}_{\varepsilon}(t, \cdot) \circ \widehat{w}^{\varepsilon}\right) \varphi\right| \leqslant C \varepsilon|\varphi|_{H^{1}(\Omega)} .
$$

So

$$
\left\|D_{t}\left(u_{\varepsilon}-\tilde{u}_{\varepsilon}(t, \cdot) \circ \widehat{w}^{\varepsilon}\right)\right\|_{\dot{H}_{\#}^{-1}(\Omega)} \leqslant C \varepsilon .
$$

This proves the desired result. 


\subsubsection{The interpolation term $D_{2}$.}

This section is devoted to the proof of the following lemma.

Lemma 10. Let $u_{\varepsilon}$ be the solution of problem (3.1) and $\tilde{u}_{\varepsilon}$ verifying (2.5). There exists a constant $C>0$ which does not depend on $\varepsilon$ and $H$ such that

$$
\left\|D_{t}\left(\left(\tilde{u}_{\varepsilon}-\pi_{H} \tilde{u}_{\varepsilon}\right) \circ \widehat{w}^{\varepsilon}\right)\right\|_{L^{2}\left((0, T), \dot{H}_{\#}^{-1}(\Omega)\right)} \leqslant C\left(H^{k}+H^{k+1} \frac{\left|b^{*}\right|}{\varepsilon}\right),
$$

where $\pi_{H}$ is the interpolation operator on $V_{H}$.

Proof. The proof of this lemma require the following lemma proved in [11].

Lemma 11. Let $g: Y \rightarrow \mathbb{R}^{N}$ be a piecewise $\mathcal{C}^{1}$ function with $\mathcal{C}^{2}$ interfaces of discontinuity such that $\int_{Y} g(y) d y=0$ and $\operatorname{div}(g)=0$. There exists a function $\zeta \in L_{\#}^{\infty}(Y)^{N \times N}$ skew-symmetric such that $g=\operatorname{div}(\zeta)$. The matrix $\zeta$ can be defined by

$$
\zeta_{i, j}=\Delta^{-1}\left(\frac{\partial g_{i}}{\partial x_{j}}-\frac{\partial g_{j}}{\partial x_{i}}\right) .
$$

Let us now break up the $D_{2}$ into three parts:

$$
\begin{aligned}
D_{t}\left(u\left(t, \widehat{w}^{\varepsilon}(x)-\frac{b^{*} t}{\varepsilon}\right)\right. & \left.-\sum_{l \in \mathcal{N}_{\mathbb{P}_{k}, H}} u\left(t, l-\frac{b^{*} t}{\varepsilon}\right) \Phi_{l}\left(\widehat{w}^{\varepsilon}(x)\right)\right) \\
=\rho^{\varepsilon}(x)\left(\partial_{t} u\left(t, \cdot-\frac{b^{*} t}{\varepsilon}\right)-\pi_{H}\left(\partial_{t} u\left(t, \cdot-\frac{b^{*} t}{\varepsilon}\right)\right)\right) \circ \widehat{w}^{\varepsilon}(x) & \\
& -\frac{1}{\varepsilon} \rho^{\varepsilon}(x) b^{*} \cdot\left(\nabla \tilde{u}_{\varepsilon}-\pi_{H} \nabla \tilde{u}_{\varepsilon}\right) \circ \widehat{w}^{\varepsilon}(x) \\
& +\frac{1}{\varepsilon} \operatorname{div}\left(b^{\varepsilon}(x)\left(\tilde{u}_{\varepsilon}-\pi_{H} \tilde{u}_{\varepsilon}\right) \circ \widehat{w}^{\varepsilon}(x)\right) .
\end{aligned}
$$

Using Remark 5 , only the $L^{2}$ norm of the first term will be bounded:

$$
\begin{aligned}
& \left\|\rho^{\varepsilon}(x)\left(\partial_{t} u\left(t, \cdot-\frac{b^{*} t}{\varepsilon}\right)-\pi_{H}\left(\partial_{t} u\left(t, \cdot-\frac{b^{*} t}{\varepsilon}\right)\right)\right) \circ \widehat{w}^{\varepsilon}\right\|_{L^{2}(\Omega)} \\
& \leqslant|\Omega|^{\frac{1}{2}} \rho_{\max }\left\|\partial_{t} u\left(t, \cdot-\frac{b^{*} t}{\varepsilon}\right)-\pi_{H}\left(\partial_{t} u\left(t, \cdot-\frac{b^{*} t}{\varepsilon}\right)\right)\right\|_{L^{\infty}(\Omega)} \\
& \leqslant C|\Omega|^{\frac{1}{2}} \rho_{\max } H^{k+1}\left\|\partial_{t} u\right\|_{W^{k+1, \infty}(\Omega)},
\end{aligned}
$$

applying, once more, interpolation results. Since

$$
\partial_{t} u=\frac{1}{\rho^{*}} \operatorname{div}\left(A^{*} \nabla u\right)
$$

we have

$$
\left\|\partial_{t} u\right\|_{W^{k+1, \infty}(\Omega)} \leqslant C\|u\|_{W^{k+3, \infty}(\Omega)} .
$$

Therefore

$$
\begin{aligned}
& \left\|\rho^{\varepsilon}(x)\left(\partial_{t} u\left(t, \cdot-\frac{b^{*} t}{\varepsilon}\right)-\pi_{H}\left(\partial_{t} u\left(t, \cdot-\frac{b^{*} t}{\varepsilon}\right)\right)\right) \circ \widehat{w}^{\varepsilon}\right\|_{\dot{H}^{-1}(\Omega)} \\
& \quad \leqslant\left\|\rho^{\varepsilon}(x)\left(\partial_{t} u\left(t, \cdot-\frac{b^{*} t}{\varepsilon}\right)-\pi_{H}\left(\partial_{t} u\left(t, \cdot-\frac{b^{*} t}{\varepsilon}\right)\right)\right) \circ \widehat{w}^{\varepsilon}\right\|_{L^{2}(\Omega)} \\
& \quad \leqslant C H^{k+1} .
\end{aligned}
$$


For the second term, let us use the inequality

$$
\left\|\left(\nabla \tilde{u}_{\varepsilon}-\pi_{H} \nabla \tilde{u}_{\varepsilon}\right) \circ \widehat{w}^{\varepsilon}\right\|_{L^{2}(\Omega)^{N}} \leqslant|\Omega|^{\frac{1}{2}}\left\|\left(\nabla \tilde{u}_{\varepsilon}-\pi_{H} \nabla \tilde{u}_{\varepsilon}\right) \circ \widehat{w}^{\varepsilon}\right\|_{L^{\infty}(\Omega)^{N}} .
$$

We have

$$
\begin{aligned}
\left\|\left(\nabla \tilde{u}_{\varepsilon}-\pi_{H} \nabla \tilde{u}_{\varepsilon}\right) \circ \widehat{w}^{\varepsilon}\right\|_{L^{\infty}(\Omega)^{N}} & =\left\|\nabla \tilde{u}_{\varepsilon}-\pi_{H} \nabla \tilde{u}_{\varepsilon}\right\|_{L^{\infty}(\Omega)^{N}} \\
& \leqslant C H^{k+1}\|\nabla u\|_{W^{k+1, \infty}(\Omega)^{N}}
\end{aligned}
$$

which gives

$$
\left\|\frac{1}{\varepsilon} \rho^{\varepsilon}(x) b^{*} \cdot\left(\nabla \tilde{u}_{\varepsilon}-\pi_{H} \nabla \tilde{u}_{\varepsilon}\right) \circ \widehat{w}^{\varepsilon}\right\|_{L^{2}(\Omega)} \leqslant C H^{k+1} \frac{\left|b^{*}\right|}{\varepsilon}\|u\|_{W^{k+2, \infty}(\Omega)}
$$

For the third term, let us define a test function $\varphi \in \dot{H}_{\#}^{1}(\Omega)$. All the functions are $\Omega$-periodic (see Remark 2), integrations by parts give:

$$
\int_{\Omega} \frac{1}{\varepsilon} \operatorname{div}\left(b^{\varepsilon}(x)\left(\tilde{u}_{\varepsilon}-\pi_{H} \tilde{u}_{\varepsilon}\right) \circ \widehat{w}^{\varepsilon}(x)\right) \varphi(x) d x=-\int_{\Omega} \frac{1}{\varepsilon} b^{\varepsilon}(x)\left(\tilde{u}_{\varepsilon}-\pi_{H} \tilde{u}_{\varepsilon}\right) \circ \widehat{w}^{\varepsilon}(x) \cdot \nabla \varphi(x) d x .
$$

This integral is split into two:

$$
\begin{aligned}
\int_{\Omega} \frac{1}{\varepsilon} b^{\varepsilon}(x)\left(\tilde{u}_{\varepsilon}-\pi_{H} \tilde{u}_{\varepsilon}\right) \circ \widehat{w}^{\varepsilon}(x) \cdot \nabla \varphi(x) d x= & \int_{\Omega} \frac{1}{\varepsilon} b^{*}\left(\tilde{u}_{\varepsilon}-\pi_{H} \tilde{u}_{\varepsilon}\right) \circ \widehat{w}^{\varepsilon}(x) \cdot \nabla \varphi(x) d x \\
& +\int_{\Omega} \frac{1}{\varepsilon}\left(b^{\varepsilon}(x)-b^{*}\right)\left(\tilde{u}_{\varepsilon}-\pi_{H} \tilde{u}_{\varepsilon}\right) \circ \widehat{w}^{\varepsilon}(x) \cdot \nabla \varphi(x) d x .
\end{aligned}
$$

For the first integral, we have

$$
\begin{aligned}
\mid \int_{\Omega} \frac{1}{\varepsilon} b^{*}\left(\tilde{u}_{\varepsilon}-\pi_{H} \tilde{u}_{\varepsilon}\right) & \circ \widehat{w}^{\varepsilon}(x) \cdot \nabla \varphi(x) d x \mid \\
& \leqslant \frac{\left|b^{*}\right|}{\varepsilon}\left\|\left(\tilde{u}_{\varepsilon}-\pi_{H} \tilde{u}_{\varepsilon}\right) \circ \widehat{w}^{\varepsilon}\right\|_{L^{\infty}(\Omega)}|\varphi|_{H^{1}(\Omega)} \\
& \leqslant C \frac{\left|b^{*}\right|}{\varepsilon} H^{k+1}\|u\|_{L^{\infty}\left((0, T), W^{k+1, \infty}(\Omega)\right)}|\varphi|_{H^{1}(\Omega)}
\end{aligned}
$$

applying Cauchy-Schwarz inequality and interpolation results. We apply Lemma 11 to the function $b^{\varepsilon}(x)-$ $\rho^{\varepsilon}(x) b^{*}$ and we define $\zeta$ the skew-symmetric matrix thus obtained. As a result,

$$
\begin{aligned}
\int_{\Omega} \frac{1}{\varepsilon}\left(b^{\varepsilon}(x)-\rho^{\varepsilon}(x) b^{*}\right)\left(\tilde{u}_{\varepsilon}-\pi_{H} \tilde{u}_{\varepsilon}\right) \circ \widehat{w}^{\varepsilon}(x) \cdot \nabla \varphi(x) d x & \\
& =\int_{\Omega} \operatorname{div}\left(\zeta\left(\frac{x}{\varepsilon}\right)\right)\left(\tilde{u}_{\varepsilon}-\pi_{H} \tilde{u}_{\varepsilon}\right) \circ \widehat{w}^{\varepsilon}(x) \cdot \nabla \varphi(x) d x .
\end{aligned}
$$

All the functions are $\Omega$-periodic (see Remark 2), integrating by parts, we obtain

$$
\begin{aligned}
\int_{\Omega} \frac{1}{\varepsilon}\left(b^{\varepsilon}(x)-\rho^{\varepsilon}(x) b^{*}\right)\left(\tilde{u}_{\varepsilon}-\right. & \left.\pi_{H} \tilde{u}_{\varepsilon}\right) \circ \widehat{w}^{\varepsilon}(x) \cdot \nabla \varphi(x) d x \\
& =\int_{\Omega} \zeta_{i, j}\left(\frac{x}{\varepsilon}\right)\left(\partial_{x_{i}}\left(\tilde{u}_{\varepsilon}-\pi_{H} \tilde{u}_{\varepsilon}\right) \circ \widehat{w}^{\varepsilon}(x) \partial_{x_{j}} \varphi(x)\right. \\
& \left.+\left(\tilde{u}_{\varepsilon}-\pi_{H} \tilde{u}_{\varepsilon}\right) \circ \widehat{w}^{\varepsilon}(x) \partial_{x_{i}, x_{j}} \varphi(x)\right) d x .
\end{aligned}
$$


Since $\zeta$ is skew-symmetric and $\partial_{x_{i}, x_{j}} \varphi=\partial_{x_{j}, x_{i}} \varphi$, the second term in the integral is equal to zero and

$$
\begin{aligned}
\int_{\Omega} \frac{1}{\varepsilon}\left(b^{\varepsilon}(x)-\rho^{\varepsilon}(x) b^{*}\right)\left(\tilde{u}_{\varepsilon}-\pi_{H} \tilde{u}_{\varepsilon}\right) \circ \widehat{w}^{\varepsilon}(x) \cdot \nabla \varphi(x) & d x \\
& =\int_{\Omega} \zeta_{i, j}\left(\frac{x}{\varepsilon}\right) \partial_{x_{i}}\left(\left(\tilde{u}_{\varepsilon}-\pi_{H} \tilde{u}_{\varepsilon}\right) \circ \widehat{w}^{\varepsilon}(x)\right) \partial_{x_{j}} \varphi(x) d x .
\end{aligned}
$$

Moreover,

$$
\partial_{x_{i}}\left(\left(\tilde{u}_{\varepsilon}-\pi_{H} \tilde{u}_{\varepsilon}\right) \circ \widehat{w}^{\varepsilon}(x)\right)=\partial_{x_{i}} \widehat{w}_{k}^{\varepsilon}(x) \partial_{x_{k}}\left(\tilde{u}_{\varepsilon}-\pi_{H} \tilde{u}_{\varepsilon}\right) \circ \widehat{w}^{\varepsilon}(x) .
$$

Hence

$$
\begin{aligned}
\left|\partial_{x_{i}}\left(\left(\tilde{u}_{\varepsilon}-\pi_{H} \tilde{u}_{\varepsilon}\right) \circ \widehat{w}^{\varepsilon}(x)\right)\right| & \leqslant\|w\|_{W^{1, \infty}(Y)}\left\|\tilde{u}_{\varepsilon}-\pi_{H} \tilde{u}_{\varepsilon}\right\|_{W^{1, \infty}(\Omega)} \\
& \leqslant\|w\|_{W^{1, \infty}(Y)} H^{k}\|u\|_{L^{\infty}\left((0, T), W^{k+1, \infty}(\Omega)\right)} .
\end{aligned}
$$

Since $\zeta \in L^{\infty}(\Omega)^{N \times N}$, we get

$$
\left|\int_{\Omega} \zeta_{i, j}\left(\frac{x}{\varepsilon}\right) \partial_{x_{i}}\left(\left(\tilde{u}_{\varepsilon}-\pi_{H} \tilde{u}_{\varepsilon}\right) \circ \widehat{w}^{\varepsilon}(x)\right) \partial_{x_{j}} \varphi(x) d x\right| \leqslant C H^{k}|\varphi|_{H^{1}(\Omega)}
$$

Therefore

$$
\int_{\Omega} \frac{1}{\varepsilon}\left(b^{\varepsilon}(x)-\rho^{\varepsilon}(x) b^{*}\right)\left(\tilde{u}_{\varepsilon}-\pi_{H} \tilde{u}_{\varepsilon}\right) \circ \widehat{w}^{\varepsilon}(x) \cdot \nabla \varphi(x) d x \leqslant C H^{k}|\varphi|_{H^{1}(\Omega)} .
$$

Injecting inequalities (4.54) and (4.55) in (4.53) gives

$$
\begin{aligned}
\mid \int_{\Omega} \frac{1}{\varepsilon} \operatorname{div}\left(b^{\varepsilon}(x)\left(\tilde{u}_{\varepsilon}-\pi_{H} \tilde{u}_{\varepsilon}\right)\right. & \left.\circ \widehat{w}^{\varepsilon}(x)\right) \varphi(x) d x \mid \\
& =\left|\int_{\Omega} \frac{1}{\varepsilon} b^{\varepsilon}(x)\left(\tilde{u}_{\varepsilon}-\pi_{H} \tilde{u}_{\varepsilon}\right) \circ \widehat{w}^{\varepsilon}(x) \cdot \nabla \varphi(x) d x\right| \\
& \leqslant C H^{k}|\varphi|_{H^{1}(\Omega)} .
\end{aligned}
$$

Thus, collecting inequalities (4.51), (4.52) and (4.56) in (4.50) implies

$$
\left\|D_{t}\left(\left(\tilde{u}_{\varepsilon}-\pi_{H} \tilde{u}_{\varepsilon}\right) \circ \widehat{w}^{\varepsilon}\right)\right\|_{L^{\infty}\left((0, T), \dot{H}_{\#}^{-1}(\Omega)\right)} \leqslant C\left(H^{k}+H^{k+1} \frac{\left|b^{*}\right|}{\varepsilon}\right) .
$$

Then, by Cauchy-Schwarz inequality, the desired inequality is obtained.

\subsubsection{The local homogenization term $D_{3}$.}

Let us prove the following lemma.

Lemma 12. Taking the same notations as in Proposition 3, there exists a constant $C>0$ such that

$$
\left\|D_{t}\left(\pi_{H} \tilde{u}_{\varepsilon}(t, \cdot) \circ \widehat{w}^{\varepsilon}-\pi_{\varepsilon, H} \tilde{u}_{\varepsilon}\right)\right\|_{L^{2}\left((0, T), \dot{H}_{\#}^{-1}(\Omega)\right)} \leqslant C\left(\sqrt{\frac{\varepsilon}{H}}+\varepsilon \frac{\left|b^{*}\right|}{\varepsilon}\right),
$$

where $\pi_{H}$ is the interpolation operator on $V_{H}$.

Proof. The norm of

$$
D_{t}\left(\pi_{H} \tilde{u}_{\varepsilon}(t, \cdot) \circ \widehat{w}^{\varepsilon}-\pi_{\varepsilon, H} \tilde{u}_{\varepsilon}\right)=D_{t}\left(\pi_{H} \tilde{u}_{\varepsilon}(t, \cdot) \circ \widehat{w}^{\varepsilon}-\pi_{H} \tilde{u}_{\varepsilon}(t, \cdot) \circ \widetilde{w}^{\varepsilon, H}\right) .
$$


needs to be bounded. Let us introduce the function $\tilde{u}_{t, \varepsilon}$ defined by

$$
\tilde{u}_{t, \varepsilon}(t, x)=\partial_{t} u\left(t, x-\frac{b^{*} t}{\varepsilon}\right) .
$$

We have

$$
\begin{aligned}
D_{t}\left(\pi_{H} \tilde{u}_{\varepsilon}(t, \cdot) \circ \widehat{w}^{\varepsilon}-\right. & \left.\pi_{H} \tilde{u}_{\varepsilon}(t, \cdot) \circ \widetilde{w}^{\varepsilon, H}\right) \\
& =\rho^{\varepsilon}\left(\pi_{H} \tilde{u}_{t, \varepsilon}(t, \cdot) \circ \widehat{w}^{\varepsilon}-\pi_{H} \tilde{u}_{t, \varepsilon}(t, \cdot) \circ \widetilde{w}^{\varepsilon, H}\right) \\
+ & \frac{1}{\varepsilon} \operatorname{div}\left(b^{\varepsilon}\left(\pi_{H} \tilde{u}_{\varepsilon}(t, \cdot) \circ \widehat{w}^{\varepsilon}-\pi_{H} \tilde{u}_{\varepsilon}(t, \cdot) \circ \widetilde{w}^{\varepsilon, H}\right)\right) \\
& \quad-\frac{1}{\varepsilon} \rho^{\varepsilon} b^{*} \cdot\left(\left(\pi_{H} \nabla \tilde{u}_{\varepsilon}\right)(t, \cdot) \circ \widehat{w}^{\varepsilon}-\left(\pi_{H} \nabla \tilde{u}_{\varepsilon}\right)(t, \cdot) \circ \widetilde{w}^{\varepsilon, H}\right) .
\end{aligned}
$$

For the first term of this sum, let us apply once more Remark 5.

$$
\begin{aligned}
&\left\|\rho^{\varepsilon}\left(\pi_{H} \tilde{u}_{t, \varepsilon}(t, \cdot) \circ \widehat{w}^{\varepsilon}-\pi_{H} \tilde{u}_{t, \varepsilon}(t, \cdot) \circ \widetilde{w}^{\varepsilon, H}\right)\right\|_{\dot{H}_{\#}^{-1}(\Omega)}^{2} \\
& \leqslant \rho_{\max }^{2} C_{\Omega}^{2}\left\|\pi_{H} \tilde{u}_{t, \varepsilon}(t, \cdot) \circ \widehat{w}^{\varepsilon}-\pi_{H} \tilde{u}_{t, \varepsilon}(t, \cdot) \circ \widetilde{w}^{\varepsilon, H}\right\|_{L^{2}(\Omega)}^{2} \\
& \leqslant \rho_{\max }^{2} C_{\Omega}^{2} \sum_{K \in \mathcal{K}_{H}}\left\|\pi_{H} \tilde{u}_{t, \varepsilon}(t, \cdot) \circ \widehat{w}^{\varepsilon}-\pi_{H} \tilde{u}_{t, \varepsilon}(t, \cdot) \circ \widetilde{w}^{\varepsilon, H}\right\|_{L^{2}(K)}^{2} .
\end{aligned}
$$

As in Section 4.3.3, in each cell $K$, this norm will be split into two in $C_{K}$ and in complement. In $K \backslash C_{K}$, let us bound the norm:

$$
\begin{aligned}
\left\|\pi_{H} \tilde{u}_{t, \varepsilon}(t, \cdot) \circ \widehat{w}^{\varepsilon}-\pi_{H} \tilde{u}_{t, \varepsilon}(t, \cdot) \circ \widetilde{w}^{\varepsilon, H}\right\|_{L^{2}\left(K \backslash C_{K}\right)} \\
\quad \leqslant C\left\|\pi_{H} \tilde{u}_{t, \varepsilon}\right\|_{L^{\infty}((0, T) \times \Omega)} \sqrt{\varepsilon|\partial K|} \\
\quad \leqslant C \sqrt{\varepsilon H^{N-1}},
\end{aligned}
$$

because

$$
\pi_{H} \tilde{u}_{t, \varepsilon}(t, x)=\sum_{l \in \mathcal{N}_{\mathbb{P}_{k}}, H} \partial_{t} u\left(t, l-\frac{b^{*} t}{\varepsilon}\right) \Phi_{l}^{H}(x)
$$

and the functions $\Phi_{l}^{H}$ and $\partial_{t} u$ are bounded.

In $C_{K}$, all the functions are $\mathcal{C}^{\infty}$ and Taylor inequalities can be used

$$
\left\|\pi_{H} \tilde{u}_{t, \varepsilon}(t, \cdot) \circ \widehat{w}^{\varepsilon}-\pi_{H} \tilde{u}_{t, \varepsilon}(t, \cdot) \circ \widetilde{w}^{\varepsilon, H}\right\|_{L^{2}\left(C_{K}\right)} \leqslant C\left\|\nabla \pi_{H} \tilde{u}_{t, \varepsilon}\right\|_{L^{\infty}(K)^{N}}\left\|\widehat{w}^{\varepsilon}-\widetilde{w}^{\varepsilon, H}\right\|_{L^{2}(K)^{N}} .
$$

Applying interpolation inequalities

$$
\left\|\pi_{H} \tilde{u}_{t, \varepsilon}\right\|_{W^{1, \infty}(K)} \leqslant\left\|\tilde{u}_{t, \varepsilon}\right\|_{W^{1, \infty}(K)}+C H^{k}\left|\tilde{u}_{t, \varepsilon}\right|_{W^{k+1, \infty}(K)} .
$$

And $\tilde{u}_{t, \varepsilon} \in W^{k+1, \infty}(\Omega)$ since $u \in W^{k+3, \infty}(\Omega)$. Moreover $k \geqslant 1$ and $H$ is small, as a consequence $\left\|\pi_{H} \tilde{u}_{t, \varepsilon}\right\|_{W^{1, \infty}(\Omega)}$ is bounded. Thus

$$
\left\|\pi_{H} \tilde{u}_{t, \varepsilon}(t, \cdot) \circ \widehat{w}^{\varepsilon}-\pi_{H} \tilde{u}_{t, \varepsilon}(t, \cdot) \circ \widetilde{w}^{\varepsilon, H}\right\|_{L^{2}\left(C_{K}\right)} \leqslant C \varepsilon \sqrt{H^{N}} .
$$

The inequalities (4.61) and (4.60) imply

$$
\left\|\pi_{H} \tilde{u}_{t, \varepsilon}(t, \cdot) \circ \widehat{w}^{\varepsilon}-\pi_{H} \tilde{u}_{t, \varepsilon}(t, \cdot) \circ \widetilde{w}^{\varepsilon, H}\right\|_{L^{2}(K)}^{2} \leqslant C \varepsilon H^{N-1}(1+\varepsilon H) .
$$


So, using inequality (4.59)

$$
\left\|\rho^{\varepsilon}\left(\pi_{H} \tilde{u}_{t, \varepsilon}(t, \cdot) \circ \widehat{w}^{\varepsilon}-\pi_{H} \tilde{u}_{t, \varepsilon}(t, \cdot) \circ \widetilde{w}^{\varepsilon, H}\right)\right\|_{\dot{H}_{\#}^{-1}(\Omega)} \leqslant C \sqrt{\frac{\varepsilon}{H}},
$$

because the number of cells in $\mathcal{K}_{H}$ is on the order of $H^{N}$ and the product $\varepsilon H$ is bounded.

In order to bound the second term in (4.58), let us introduce a test function $\varphi \in \dot{H}_{\#}^{1}(\Omega)$. Applying Remark 2 in the integration by parts on $\Omega$ :

$$
\begin{aligned}
\int_{\Omega} \frac{1}{\varepsilon} \operatorname{div}\left(b ^ { \varepsilon } ( x ) \left(\pi_{H} \tilde{u}_{\varepsilon}\left(t, \widehat{w}^{\varepsilon}(x)\right)-\pi_{H} \tilde{u}_{\varepsilon}\right.\right. & \left.\left.\left(t, \widetilde{w}^{\varepsilon, H}\right)\right)\right) \varphi(x) d x \\
& =-\int_{\Omega} \frac{1}{\varepsilon} b^{\varepsilon}(x)\left(\pi_{H} \tilde{u}_{\varepsilon}\left(t, \widehat{w}^{\varepsilon}(x)\right)-\pi_{H} \tilde{u}_{\varepsilon}\left(t, \widetilde{w}^{\varepsilon, H}(x)\right)\right) \cdot \nabla \varphi(x) d x,
\end{aligned}
$$

the boundary term is equal to zero because the functions $b^{\varepsilon}, \pi_{H} \tilde{u}_{\varepsilon}(t, \cdot) \circ \widehat{w}^{\varepsilon}, \pi_{H} \tilde{u}_{\varepsilon}(t, \cdot) \circ \widetilde{w}^{\varepsilon, H}$ and $\varphi$ are $\Omega$-periodic. Using once more the skew-symmetric matrix

$$
\zeta \in L_{\#}^{\infty}(Y)^{N \times N}
$$

such that $b(y)-\rho(y) b^{*}=\operatorname{div}(\zeta(y))$. Then

$$
\begin{aligned}
\int_{\Omega} \frac{1}{\varepsilon} & \left(b^{\varepsilon}(x)-\rho^{\varepsilon}(x) b^{*}\right)\left(\pi_{H} \tilde{u}_{\varepsilon}\left(t, \widehat{w}^{\varepsilon}(x)\right)-\pi_{H} \tilde{u}_{\varepsilon}\left(t, \widetilde{w}^{\varepsilon, H}(x)\right)\right) \cdot \nabla \varphi(x) d x \\
& =\int_{\Omega} \operatorname{div}\left(\zeta\left(\frac{x}{\varepsilon}\right)\right)\left(\pi_{H} \tilde{u}_{\varepsilon}\left(t, \widehat{w}^{\varepsilon}(x)\right)-\pi_{H} \tilde{u}_{\varepsilon}\left(t, \widetilde{w}^{\varepsilon, H}(x)\right)\right) \cdot \nabla \varphi(x) d x \\
& =-\int_{\Omega} \zeta_{i, j}\left(\frac{x}{\varepsilon}\right) \partial_{x_{i}}\left(\left(\pi_{H} \tilde{u}_{\varepsilon}\left(t, \widehat{w}^{\varepsilon}(x)\right)-\pi_{H} \tilde{u}_{\varepsilon}\left(t, \widetilde{w}^{\varepsilon, H}(x)\right)\right) \partial_{x_{j}} \varphi(x)\right) d x
\end{aligned}
$$

the boundary terms being equal to zero due to the previous remarks. The matrix $\zeta$ being skew-symmetric, the term in $\partial_{x_{i}, x_{j}}^{2} \varphi$ is equal to zero and

$$
\begin{aligned}
\int_{\Omega} \frac{1}{\varepsilon}\left(b^{\varepsilon}(x)-\rho^{\varepsilon}(x) b^{*}\right)\left(\pi_{H} \tilde{u}_{\varepsilon}\right. & \left.\left(t, \widehat{w}^{\varepsilon}(x)\right)-\pi_{H} \tilde{u}_{\varepsilon}\left(t, \widetilde{w}^{\varepsilon, H}(x)\right)\right) \cdot \nabla \varphi(x) d x \\
& =-\int_{\Omega} \zeta_{i, j}\left(\frac{x}{\varepsilon}\right) \partial_{x_{i}}\left(\pi_{H} \tilde{u}_{\varepsilon}\left(t, \widehat{w}^{\varepsilon}(x)\right)-\pi_{H} \tilde{u}_{\varepsilon}\left(t, \widetilde{w}^{\varepsilon, H}(x)\right)\right) \partial_{x_{j}} \varphi(x) d x .
\end{aligned}
$$

Let us now write

$$
\begin{array}{r}
\partial_{x_{i}}\left(\pi_{H} \tilde{u}_{\varepsilon}\left(t, \widehat{w}^{\varepsilon}(x)\right)-\pi_{H} \tilde{u}_{\varepsilon}\left(t, \widetilde{w}^{\varepsilon, H}(x)\right)\right) \\
=\partial_{x_{i}} \widehat{w}_{k}^{\varepsilon}(x) \partial_{x_{k}} \pi_{H} \tilde{u}_{\varepsilon}\left(t, \widehat{w}^{\varepsilon}(x)\right)-\partial_{x_{i}} \widetilde{w}_{k}^{\varepsilon, H}(x) \partial_{x_{k}} \pi_{H} \tilde{u}_{\varepsilon}\left(t, \widetilde{w}^{\varepsilon, H}(x)\right) \\
=\left(\partial_{x_{i}} \widehat{w}_{k}^{\varepsilon}(x)-\partial_{x_{i}} \widetilde{w}_{k}^{\varepsilon, H}(x)\right) \partial_{x_{k}} \pi_{H} \tilde{u}_{\varepsilon}\left(t, \widetilde{w}^{\varepsilon, H}(x)\right) \\
\quad+\partial_{x_{i}} \widehat{w}_{k}^{\varepsilon}(x)\left(\partial_{x_{k}} \pi_{H} \tilde{u}_{\varepsilon}\left(t, \widehat{w}^{\varepsilon}(x)\right)-\partial_{x_{k}} \pi_{H} \tilde{u}_{\varepsilon}\left(t, \widetilde{w}^{\varepsilon, H}(x)\right)\right) .
\end{array}
$$


Applying computations done previously:

$$
\begin{aligned}
\|\left(\partial_{x_{i}} \widehat{w}_{k}^{\varepsilon}-\partial_{x_{i}} \widetilde{w}_{k}^{\varepsilon, H}\right) & \partial_{x_{k}} \pi_{H} \tilde{u}_{\varepsilon}(t, \cdot) \circ \widetilde{w}^{\varepsilon, H} \|_{L^{2}(\Omega)}^{2} \\
& =\sum_{K \in \mathcal{K}_{H}}\left\|\left(\partial_{x_{i}} \widehat{w}_{k}^{\varepsilon}-\partial_{x_{i}} \widetilde{w}_{k}^{\varepsilon, H}\right) \partial_{x_{k}} \pi_{H} \tilde{u}_{\varepsilon}(t, \cdot) \circ \widetilde{w}^{\varepsilon, H}\right\|_{L^{2}(K)}^{2} \\
& \leqslant \sum_{K \in \mathcal{K}_{H}}\left\|\nabla \widehat{w}^{\varepsilon}-\nabla \widetilde{w}^{\varepsilon, H}\right\|_{L^{2}(K)^{N \times N}}^{2}\left\|\nabla \pi_{H} \tilde{u}_{\varepsilon}\right\|_{L^{\infty}(\Omega)^{N}}^{2} \\
& \leqslant C \sum_{K \in \mathcal{K}_{H}} \varepsilon H^{N-1} \\
& \leqslant C \frac{\varepsilon}{H} .
\end{aligned}
$$

Moreover, a Taylor expansion can be applied in the set $C_{K}$ :

$$
\begin{aligned}
\| \partial_{x_{i}} \widehat{w}_{k}^{\varepsilon}\left(\partial_{x_{k}} \pi_{H} \tilde{u}_{\varepsilon}(t, \cdot) \circ \widehat{w}^{\varepsilon}\right. & \left.-\partial_{x_{k}} \pi_{H} \tilde{u}_{\varepsilon}(t, \cdot) \circ \widetilde{w}^{\varepsilon, H}\right) \|_{L^{2}\left(C_{K}\right)} \\
& \leqslant\left\|\widehat{w}^{\varepsilon}\right\|_{W^{1, \infty}(\Omega)^{N}}\left\|\widehat{w}^{\varepsilon}-\widetilde{w}^{\varepsilon, H}\right\|_{L^{2}(K)^{N}}\left\|\nabla^{2} \pi_{H} \tilde{u}_{\varepsilon}\right\|_{L^{\infty}(K)^{N}} \\
& \leqslant C \varepsilon \sqrt{H^{N}} .
\end{aligned}
$$

In $K \backslash C_{K}$, since $\left|K \backslash C_{K}\right| \leqslant C|\partial K| \varepsilon$, we have

$$
\begin{aligned}
\| \partial_{x_{i}} \widehat{w}_{k}^{\varepsilon}\left(\partial_{x_{k}} \pi_{H} \tilde{u}_{\varepsilon}(t, \cdot) \circ \widehat{w}^{\varepsilon}-\right. & \left.\partial_{x_{k}} \pi_{H} \tilde{u}_{\varepsilon}(t, \cdot) \circ \widetilde{w}^{\varepsilon, H}\right) \|_{L^{2}\left(K \backslash C_{K}\right)} \\
& \leqslant 2\left\|\widehat{w}^{\varepsilon}\right\|_{W^{1, \infty}(\Omega)}\left\|\nabla \pi_{H} \tilde{u}_{\varepsilon}\right\|_{L^{\infty}(K)^{N \times N}} \sqrt{C|\partial K| \varepsilon} \\
& \leqslant C \sqrt{\varepsilon H^{N-1}} .
\end{aligned}
$$

Hence, gathering inequalities (4.67) and (4.68):

$$
\left\|\partial_{x_{i}} \widehat{w}_{k}^{\varepsilon}\left(\partial_{x_{k}} \pi_{H} \tilde{u}_{\varepsilon}(t, \cdot) \circ \widehat{w}^{\varepsilon}-\partial_{x_{k}} \pi_{H} \tilde{u}_{\varepsilon}(t, \cdot) \circ \widetilde{w}^{\varepsilon, H}\right)\right\|_{L^{2}(K)} \leqslant C \sqrt{\varepsilon H^{N-1}} .
$$

Therefore

$$
\begin{aligned}
\left\|\partial_{x_{i}} \widehat{w}_{k}^{\varepsilon}\left(\partial_{x_{k}} \pi_{H} \tilde{u}_{\varepsilon}(t, \cdot) \circ \widehat{w}^{\varepsilon}-\partial_{x_{k}} \pi_{H} \tilde{u}_{\varepsilon}(t, \cdot) \circ \widetilde{w}^{\varepsilon, H}\right)\right\|_{L^{2}(\Omega)}^{2} \\
\quad=\sum_{K \in \mathcal{K}_{H}}\left\|\partial_{x_{i}} \widehat{w}_{k}^{\varepsilon}\left(\partial_{x_{k}} \pi_{H} \tilde{u}_{\varepsilon}(t, \cdot) \circ \widehat{w}^{\varepsilon}-\partial_{x_{k}} \pi_{H} \tilde{u}_{\varepsilon}(t, \cdot) \circ \widetilde{w}^{\varepsilon, H}\right)\right\|_{L^{2}(K)}^{2} \\
\quad \leqslant \sum_{K \in \mathcal{K}_{H}} C \varepsilon H^{N-1} \\
\quad \leqslant C \frac{\varepsilon}{H}
\end{aligned}
$$

Injecting inequalities (4.66) and (4.69) in (4.64) gives

$$
\left\|\partial_{x_{i}}\left(\pi_{H} \tilde{u}_{\varepsilon}\left(t, \widehat{w}^{\varepsilon}(x)\right)-\pi_{H} \tilde{u}_{\varepsilon}\left(t, \widetilde{w}^{\varepsilon, H}(x)\right)\right)\right\|_{L^{2}(\Omega)} \leqslant C \sqrt{\frac{\varepsilon}{H}} .
$$

And, using inequality (4.63) and Cauchy-Schwarz inequality leads to

$$
\begin{aligned}
\left|\int_{\Omega} \frac{1}{\varepsilon}\left(b^{\varepsilon}(x)-\rho^{\varepsilon}(x) b^{*}\right)\left(\pi_{H} \tilde{u}_{\varepsilon}\left(t, \widehat{w}^{\varepsilon}(x)\right)-\pi_{H} \tilde{u}_{\varepsilon}\left(t, \widetilde{w}^{\varepsilon, H}(x)\right)\right) \cdot \nabla \varphi(x) d x\right| & \\
& \leqslant C|\varphi|_{H^{1}(\Omega)} \sqrt{\frac{\varepsilon}{H}} .
\end{aligned}
$$


$\pi_{H} \tilde{u}_{\varepsilon}$ is bounded in $W^{1, \infty}(\Omega)$, thus we get

$$
\left\|\pi_{H} \tilde{u}_{\varepsilon}(t, \cdot) \circ \widehat{w}^{\varepsilon}-\pi_{H} \tilde{u}_{\varepsilon}(t, \cdot) \circ \widetilde{w}^{\varepsilon, H}\right\|_{L^{2}(K)} \leqslant C\left\|\widehat{w}^{\varepsilon}-\widetilde{w}^{\varepsilon, H}\right\|_{L^{2}(K)} .
$$

Then, applying Lemma 6, we have

$$
\left\|\pi_{H} \tilde{u}_{\varepsilon}(t, \cdot) \circ \widehat{w}^{\varepsilon}-\pi_{H} \tilde{u}_{\varepsilon}(t, \cdot) \circ \widetilde{w}^{\varepsilon, H}\right\|_{L^{2}(K)} \leqslant C C \varepsilon \sqrt{|K|} .
$$

This implies

$$
\begin{aligned}
\left\|\pi_{H} \tilde{u}_{\varepsilon}(t, \cdot) \circ \widehat{w}^{\varepsilon}-\pi_{H} \tilde{u}_{\varepsilon}(t, \cdot) \circ \widetilde{w}^{\varepsilon, H}\right\|_{L^{2}(\Omega)}^{2} & \\
& =\sum_{K \in \mathcal{K}_{H}}\left\|\pi_{H} \tilde{u}_{\varepsilon}(t, \cdot) \circ \widehat{w}^{\varepsilon}-\pi_{H} \tilde{u}_{\varepsilon}(t, \cdot) \circ \widetilde{w}^{\varepsilon, H}\right\|_{L^{2}(K)}^{2} \\
& \leqslant C \varepsilon^{2} \sum_{K \in \mathcal{K}_{H}}|K| \\
& \leqslant C|\Omega| \varepsilon^{2} .
\end{aligned}
$$

Therefore, applying Cauchy-Schwarz inequality

$$
\left|\int_{\Omega} \frac{1}{\varepsilon} \rho^{\varepsilon}(x) b^{*}\left(\pi_{H} \tilde{u}_{\varepsilon}\left(t, \widehat{w}^{\varepsilon}(x)\right)-\pi_{H} \tilde{u}_{\varepsilon}\left(t, \widetilde{w}^{\varepsilon, H}(x)\right)\right) \cdot \nabla \varphi(x) d x\right| \leqslant C \frac{\left|b^{*}\right|}{\varepsilon} \varepsilon|\varphi|_{H^{1}(\Omega)} .
$$

And, collecting inequalities (4.71) and (4.70):

$$
\left|\int_{\Omega} \frac{1}{\varepsilon} b^{\varepsilon}(x)\left(\pi_{H} \tilde{u}_{\varepsilon}\left(t, \widehat{w}^{\varepsilon}(x)\right)-\pi_{H} \tilde{u}_{\varepsilon}\left(t, \widetilde{w}^{\varepsilon, H}(x)\right)\right) \cdot \nabla \varphi(x) d x\right| \leqslant C\left(\sqrt{\frac{\varepsilon}{H}}+\frac{\left|b^{*}\right|}{\varepsilon} \varepsilon\right)|\varphi|_{H^{1}(\Omega)} .
$$

Moreover, using the same arguments that led to inequality (4.71), we have

$$
\left|\int_{\Omega} \frac{1}{\varepsilon} \rho^{\varepsilon}(x) b^{*} \cdot\left(\pi_{H} \nabla \tilde{u}_{\varepsilon}\left(t, \widehat{w}^{\varepsilon}(x)\right)-\pi_{H} \nabla \tilde{u}_{\varepsilon}\left(t, \widetilde{w}^{\varepsilon, H}(x)\right)\right) \varphi(x) d x\right| \leqslant C \frac{\left|b^{*}\right|}{\varepsilon} \varepsilon\|\varphi\|_{L^{2}(\Omega)} .
$$

Applying inequalities (4.62), (4.72) and (4.73) in (4.58), we get

$$
\left\|D_{t}\left(\pi_{H} \tilde{u}_{\varepsilon}(t, \cdot) \circ \widehat{w}^{\varepsilon}-\pi_{\varepsilon, H} \tilde{u}_{\varepsilon}\right)\right\|_{\dot{H}_{\#}^{-1}(\Omega)} \leqslant C\left(\sqrt{\frac{\varepsilon}{H}}+\frac{\left|b^{*}\right|}{\varepsilon} \varepsilon\right) .
$$

This proves the desired result. $\square$

\section{Acknowledgement.}

The results presented in this paper are part of F. Ouaki's PhD work which was supported by IFP Energies nouvelles.

\section{REFERENCES}

[1] G. Allaire, R. Brizzi, A. Mikelić and A.L. Piatnitski. Two-scale expansion with drift approach to the Taylor dispersion for reactive transport through porous media. Chem. Eng. Sci., 65(7):2292-2300, 2010.

[2] G. Allaire and R. Brizzi. A multiscale finite element method for numerical homogenization. Multiscale Modeling and Simulation, 4(3):125-144, 2006.

[3] G. Allaire, S. Desroziers, G. Enchéry and F. Ouaki. A Multiscale Finite Element Method for Transport Modeling. Proceedings of the 6th European Congress on Computational Methods in Applied Sciences and Engineering (ECCOMAS 2012), September 10-14, 2012, Vienna, Austria. 
[4] G. Allaire, A. Mikelić and A.L. Piatnitski, Homogenization approach to the dispersion theory for reactive transport through porous media. SIAM J. Math. Anal., 42(1):125-144, 2010.

[5] G. Allaire, I. Pankratova, A. Piatnitski. Homogenization and concentration for a diffusion equation with large convection in a bounded domain. Journal of Functional Analysis, 262(1):300-330, 2012.

[6] G. Allaire and A.L. Raphael. Homogenization of a convection-diffusion model with reaction in a porous medium. $C$. $R$. Math. Acad. Sci. Paris, 344(8):523-528, 2007.

[7] T. Arbogast. Numerical subgrid upscaling of two-phase flow in porous media. Lecture Notes in Physics (Z. Chen, R. Ewing, and Z.-C. Shi, eds.), 35-49, Springer-Verlag, Berlin, 2000.

[8] A. Bensoussan, J.L. Lions and G. Papanicolaou. Asymptotic analysis for periodic structures. North Holland, Amsterdam, 1972.

[9] M. Bercovier, O. Pironneau and V. Sastri. Finite elements and characteristics for some parabolic-hyperbolic problems. Appl. Math. Modelling, 7(2):89-96, 1983.

[10] A.N. Brooks and Th.J.R. Hughes. Streamline upwind/petrov-galerkin formulations for convection dominated flows with particular emphasis on the incompressible navier-stokes equations. Comput. Methods Appl. Mech. Eng., 32(1):199-259, 1982.

[11] Y. Capdeboscq. Homogenization of a diffusion equation with drift. C. R. Acad. Sci., Paris, Sér. I, Math., 327(9):807-812, 1998.

[12] J. Céa. Approximation variationnelle des problèmes aux limites. Ann. Inst. Fourier, 14(2):345-444, 1964.

[13] P. G. Ciarlet. The finite element method for elliptic problems. North-Holland, 1978.

[14] P. Donato and A.L. Piatnitski. Averaging of nonstationary parabolic operators with large lower order terms. Multi Scale Problems and Asymptotic Analysis. GAKUTO Internat. Ser. Math. Sci. Appl, 24:153-165, 2005.

[15] W. E and B. Engquist. The heterogeneous multiscale methods. Comm. Math. Sci., 1(1):87-132, 2003.

[16] Y. Efendiev and Th.Y. Hou. Multiscale finite element methods: theory and applications. Springer Verlag, 2009.

[17] P. Henning and M. Ohlberger. The heterogeneous multiscale finite element method for advection-diffusion problems with rapidly oscillating coefficients and large expected drift. Netw. Heterog. Media, 5(4):711-744, 2010.

[18] Th.Y. Hou and D. Liang. Multiscale analysis for convection dominated transport equations. Discrete Contin. Dyn. Syst., 23(1-2):281-298, 2009.

[19] Th.Y. Hou and X.H. Wu. A multiscale finite element method for elliptic problems in composite materials and porous media. J. Comput. Phys., 134(1):169-189, 1997.

[20] Th. Y. Hou, X.-H. Wu and Z. Cai. Convergence of a multiscale finite element method for elliptic problems with rapidly oscillating coefficients. Math. Comput., 68(227):913-943, 1999.

[21] S. M. Kozlov. Averaging of random operators. Math. USSR-Sb., 37(2):167-180, 1980.

[22] E. Marušić-Paloka and A.L. Piatnitski. Homogenization of a nonlinear convection-diffusion equation with rapidly oscillating coefficients and strong convection, J. London Math. Soc., 72(2):391-409, 2005.

[23] A.M. Matache, I. Babuška and C. Schwab. Generalized p-fem in homogenization. Numer. Math., 86(2):319-375, 2000.

[24] F. Ouaki. Études de méthodes multi-échelles pour la simulation de réservoir, PhD Thesis, École Polytechnique, 2013. http://tel.archives-ouvertes.fr/docs/00/92/27/83/PDF/thesis-report.pdf

[25] H. Owhadi and L. Zhang. Metric-based upscaling. Commun. Pure Appl. Math., 60(5):675-723, 2007.

[26] G. C. Papanicolaou, Diffusion in random media. Surv. Appl. Math., 1:205-253, 1995.

[27] O. Pironneau, J. Liou and T. Tezduyar. Characteristic-galerkin and galerkin/least-squares space-time formulations for the advection-diffusion equation with time-dependent domains, Comput. Methods Appl. Mech. Eng., 100(1):117-141, 1992.

[28] É. Sanchez-Palencia. Non-homogeneous media and vibration theory. Springer-Verlag, 1980.

[29] L. Tartar. The general theory of homogenization : a personalized introduction, 7. Springer, 2009.

[30] M. F. Wheeler. A priori $\mathrm{L}_{2}$ error estimates for Galerkin approximations to parabolic partial differential equations, SIAM J. Numer. Anal., 10(4):723-759, 1973.

[31] V.V. Zhikov, S.M. Kozlov and O.A. Oleinik. Homogenization of differential operators and integral functionals. SpringerVerlag, 1994. 